\title{
Nielsen coincidence numbers, Hopf invariants and spherical space forms
}

\author{
ULRICH KOSCHORKE
}

\begin{abstract}
Given two maps between smooth manifolds, the obstruction to removing their coincidences (via homotopies) is measured by the minimum numbers. In order to determine them we introduce and study an infinite hierarchy of Nielsen numbers $N_{i}, i=0,1, \ldots, \infty$. They approximate the minimum numbers from below with decreasing accuracy, but they are (in principle) more easily computable as $i$ grows. If the domain and the target manifold have the same dimension (eg in the fixed point setting) all these Nielsen numbers agree with the classical definition. However, in general they can be quite distinct.

While our approach is very geometric, the computations use the techniques of homotopy theory and, in particular, all versions of Hopf invariants (à la Ganea, Hilton or James). As an illustration we determine all Nielsen numbers and minimum numbers for pairs of maps from spheres to spherical space forms. Maps into even dimensional real projective spaces turn out to produce particularly interesting coincidence phenomena.
\end{abstract}

55M20; 55Q25, 55Q40

Dedicated to Karl-Otto Stöhr on the occasion of his $70^{\text {th }}$ birthday.

\section{Introduction}

Consider (continuous) maps $f_{1}, f_{2}: X \rightarrow Y$ between connected smooth manifolds without boundary, of dimensions $m$ and $n$, resp., $X$ being compact. We are interested in "essential" aspects of their coincidence set

$$
C=C\left(f_{1}, f_{2}\right):=\left\{x \in X \mid f_{1}(x)=f_{2}(x)\right\},
$$

ie in those features which are preserved by homotopies $f_{i} \sim f_{i}^{\prime}, i=1,2$. Such essential phenomena can be measured to some extent by the minimum numbers (of coincidence points and path-components, resp.)

$$
\operatorname{MC}\left(f_{1}, f_{2}\right):=\min \left\{\# C\left(f_{1}^{\prime}, f_{2}^{\prime}\right) \mid f_{1}^{\prime} \sim f_{1}, f_{2}^{\prime} \sim f_{2}\right\}
$$


and (even better)

$$
\operatorname{MCC}\left(f_{1}, f_{2}\right):=\min \left\{\# \pi_{0}\left(C\left(f_{1}^{\prime}, f_{2}^{\prime}\right)\right) \mid f_{1}^{\prime} \sim f_{1}, f_{2}^{\prime} \sim f_{2}\right\} .
$$

Eg both numbers vanish precisely if the maps $f_{1}, f_{2}$ can be deformed until they are coincidence free; in this case we say that the pair $\left(f_{1}, f_{2}\right)$ is loose.

Example 1.4 (Topological fixed point theory) Here $X=Y, f_{2}=\mathrm{id}_{X}$, and the principal object of study is the minimum number of fixed points

$$
\operatorname{MF}(f):=\min \left\{\# C\left(f^{\prime}, \operatorname{id}_{X}\right) \mid f^{\prime} \sim f\right\}=\operatorname{MC}\left(f, \operatorname{id}_{X}\right)
$$

for maps $f: X \rightarrow X$ (cf Brown [3, page 9]; see also Brooks [2]). If it vanishes then so does the Lefschetz number, but the converse conclusion fails to hold in general. A powerful tool for a better understanding of minimum numbers was introduced by Jakob Nielsen in the 1920s when he described a lower bound $N(f)$ of $\operatorname{MC}\left(f, \mathrm{id}_{X}\right)$. This "Nielsen number" turned out to coincide with the minimum fixed point number precisely when $X$ is not a surface with strictly negative Euler characteristic. (For an account of the spectacular history of this result see [3].)

In general coincidence theory, the geometry of generic coincidence phenomena, is much richer. Eg, when $X^{m}, Y^{n}$ are smooth manifolds of dimensions $m>n$, then $C$ is generically an $(m-n)$-manifold (and not just a finite set of isolated points).

In this paper we introduce an infinite hierarchy of (integer) Nielsen coincidence numbers which are lower bounds for the minimum numbers:

$$
(\mathrm{MC} \geq \mathrm{MCC} \geq) N^{\#} \equiv N_{0} \geq N_{1} \geq N_{2} \geq \cdots \geq N_{r} \geq \cdots \geq N_{\infty} \equiv \widetilde{N} \geq 0
$$

This hierarchy interpolates between the sharpest ("nonstabilized") Nielsen number $N_{0}:=N^{\#}$ introduced in Koschorke [11] and the ("fully stabilized") Nielsen number $N_{\infty}:=\widetilde{N}$ (cf Koschorke [14]; $\widetilde{N}$ was introduced and discussed originally under the name $N$ in Koschorke [10] and also in Koschorke [11; 12; 13]).

For every pair $f_{1}, f_{2}: X \rightarrow Y$ of maps and $r=0,1,2, \ldots, \infty$, the Nielsen number $N_{r}\left(f_{1}, f_{2}\right)=N_{r}\left(f_{2}, f_{1}\right) \in \mathbb{Z}$ depends only on the homotopy classes of $f_{1}$ and $f_{2}$. It is extracted from the bordism class

$$
\omega_{r}\left(f_{1}, f_{2}\right)=\left[\left(i_{r}: C\left(f_{1}^{\prime}, f_{2}^{\prime}\right) \subset X \times \mathbb{R}^{r}, \tilde{g}, \bar{g}_{r}\right)\right]
$$

which captures the geometric coincidence data of a generic pair $\left(f_{1}^{\prime}, f_{2}^{\prime}\right)$ approximating $\left(f_{1}, f_{2}\right)$ : the vector bundle isomorphism

$$
\bar{g}_{r}:=\bar{g}^{\#} \times \mathrm{id}: v\left(i_{r}\right)=v\left(i_{0}\right) \times \mathbb{R}^{r} \cong f_{1}^{*}(T Y) \times \mathbb{R}^{r}
$$


(compare $[11,(13)])$ describes the normal bundle $v\left(i_{r}\right)$ of the coincidence set $C\left(f_{1}^{\prime}, f_{2}^{\prime}\right)$, considered as a submanifold of $X \times \mathbb{R}^{r}$, and the map

$$
\tilde{g}: C\left(f_{1}^{\prime}, f_{2}^{\prime}\right) \rightarrow E\left(f_{1}, f_{2}\right)
$$

into the so-called homotopy coincidence space $E\left(f_{1}, f_{2}\right)$ of $\left(f_{1}, f_{2}\right)$ involves the constant paths at $f_{1}^{\prime}(x)=f_{2}^{\prime}(x), x \in C\left(f_{1}^{\prime}, f_{2}^{\prime}\right)$ (for details see [10, Section 2, (3.2) and $(4.2) ; 11,1.6])$.

The decomposition of $E\left(f_{1}, f_{2}\right)$ into its path-components induces the Nielsen decomposition

$$
C\left(f_{1}^{\prime}, f_{2}^{\prime}\right)=\coprod_{A \in \pi_{0}\left(E\left(f_{1}, f_{2}\right)\right)} \tilde{g}^{-1}(A)
$$

Given $r$, we call a path-component $A$ of $E\left(f_{1}, f_{2}\right)$ essential if the corresponding triple

$$
\left(i_{r}\left|: \tilde{g}^{-1}(A) \subset X \times \mathbb{R}^{r}, \tilde{g}\right|, \bar{g}_{r} \mid\right)
$$

of restricted coincidence data is nullbordant. Define $N_{r}\left(f_{1}, f_{2}\right)$ to be the number of these essential path-components. (For more details compare $[10 ; 11 ; 14]$, as well as Sections 2 and 3 below.) Clearly $N_{r}\left(f_{1}, f_{2}\right)$ vanishes if $\left(f_{1}, f_{2}\right)$ is loose (ie, homotopic to a coincidence free pair).

In the "stable" dimension range $m<2 n-2$, the Nielsen numbers $N_{r}\left(f_{1}, f_{2}\right)$ are independent of $r$ (and agree even with $\operatorname{MCC}\left(f_{1}, f_{2}\right) ; \operatorname{cf}$ [10, Theorem 1.10]). Moreover in the setting of fixed point theory our Nielsen numbers $N_{r}$ (cf (1.5)) all coincide with the classical Nielsen number. However, in general they can be quite distinct: often they get weaker but also more easily computable as $r$ increases (see eg Example 1.15 below).

The following 2-step program suggests itself.

(I) Decide when $\operatorname{MCC}\left(f_{1}, f_{2}\right)$ is equal to a Nielsen number $N_{r}\left(f_{1}, f_{2}\right)$ (and for which $r$ ). In topological fixed point theory this was the central unsolved problem for nearly 60 years. In general coincidence theory, complete answers have been given only in some simple settings; often they involve deep notions of differential topology and homotopy theory such as Kervaire invariants, all versions of Hopf invariants or the elements in the stable homotopy of spheres defined by invariantly framed Lie groups (cf Koschorke [14] and Koschorke and Randall [16]).

(II) Determine the Nielsen numbers $N_{r}\left(f_{1}, f_{2}\right)$. 
In this paper we concentrate our attention mainly on this step (II) and on the important special case when the domain of the maps $f_{1}, f_{2}$ is a sphere. Again all types of Hopf invariants (à la Ganea, Hilton, James) turn out to play an important role.

We need some preliminary explanations. Choose an oriented compact $n$-dimensional ball $B$ (with boundary sphere $\partial B$ ), embedded in the universal covering space $\tilde{Y}$ of $Y$. Let

$$
b: \tilde{Y} \rightarrow \tilde{Y} /(\tilde{Y}-\stackrel{\circ}{B})=B / \partial B \cong S^{n}
$$

denote the collapsing map. Moreover let

$$
H_{\mathcal{C}}:\left[S^{m}, \tilde{Y}\right] \cong \pi_{m}(\tilde{Y}) \rightarrow \pi_{m}\left(S^{n} b \tilde{Y}\right)
$$

be the Hopf-Ganea invariant homomorphism based on the cofibration

$$
\mathcal{C}: \partial B \subset \tilde{Y} \backslash \stackrel{\circ}{B} \rightarrow \tilde{Y}
$$

(cf Cornea, Lupton, Oprea and Tanré [6, 6.44 and 6.45]); here $S^{n}$ b $\tilde{Y}$ denotes the homotopy fiber of the inclusion of the one-point union $S^{n} \vee \tilde{Y}$ into $S^{n} \times \tilde{Y}$ (cf Ganea [7, (9)] and Cornea, Lupton, Oprea and Tanré [6, Section 6.7]).

In the appendix of this paper we present and use an explicit geometric description of partial suspension homomorphisms

$$
e^{r}: \pi_{m}\left(S^{n} b \tilde{Y}\right) \rightarrow \pi_{m+r}\left(S^{n+r_{b}} \tilde{Y}\right), \quad r=0,1,2, \ldots, \infty,
$$

very closely related to those discussed by H J Baues (compare [1, Chapter 3]).

Theorem 1.11 Let $Y$ be a connected smooth $n$-dimensional manifold without boundary and write $k:=\# \pi_{1}(Y)$ for the order of the fundamental group. Also let $0 \leq r \leq \infty$ and assume $m \geq 2$. Given $\left[f_{1}\right],\left[f_{2}\right] \in \pi_{m}\left(Y, y_{0}\right)$, let $\left[\tilde{f}_{1}\right],\left[\tilde{f}_{2}\right] \in \pi_{m}\left(\tilde{Y}, \tilde{y}_{0}\right)$ be liftings to the universal covering space $\widetilde{Y}$ of $Y$.

Case $1 \pi_{1}(Y)$ is infinite or $Y$ is not compact or $\boldsymbol{m}<n \quad$ Then $\operatorname{MCC}\left(f_{1}, f_{2}\right)=0$ and all Nielsen numbers vanish.

Case $2 \mathbf{2} \leq \boldsymbol{k}<\infty$ Choose a map $a^{\bullet}:\left(\tilde{Y}, \tilde{y}_{0}\right) \rightarrow\left(\tilde{Y}, \tilde{y}_{0}\right)$ that is freely homotopic to a fixed point free selfmap $a$ of $\tilde{Y}$ (eg to a covering transformation $a \neq$ identity map). Then precisely one of the following four conditions holds (compare (1.8) and (1.9)):

$$
\begin{array}{rlll}
\left(*_{k}\right) & H_{\mathcal{C}}\left(\tilde{f}_{1}\right) \neq H_{\mathcal{C}}\left(\tilde{f}_{2}\right) & \text { or } & b \circ \tilde{f}_{1} \not b \circ \tilde{f}_{2} \not b \circ a \circ \tilde{f}_{1} \\
\left(*_{k-1}\right) & H_{\mathcal{C}}\left(\tilde{f}_{1}\right)=H_{\mathcal{C}}\left(\tilde{f}_{2}\right) & \text { and } & b \circ \tilde{f}_{1} \not b \circ a \circ \tilde{f}_{1} \sim b \circ \tilde{f}_{2} \\
\left(*_{1}\right) & H_{\mathcal{C}}\left(\tilde{f}_{1}\right)=H_{\mathcal{C}}\left(\tilde{f}_{2}\right) & \text { and } & b \circ a \circ \tilde{f}_{1} \not b \circ \tilde{f}_{1} \sim b \circ \tilde{f}_{2} \\
\left(*_{0}\right) & H_{\mathcal{C}}\left(\tilde{f}_{1}\right)=H_{\mathcal{C}}\left(\tilde{f}_{2}\right) & \text { and } & b \circ a \circ \tilde{f}_{1} \sim b \circ \tilde{f}_{1} \sim b \circ \tilde{f}_{2}
\end{array}
$$


If the condition $\left(*_{i}\right)$ is satisfied for $i=0,1, k-1$ or $k$, then

$$
N^{\#}\left(f_{1}, f_{2}\right)=i \text {. }
$$

Precisely the analogous result holds for $N_{r}\left(f_{1}, f_{2}\right), r=0,1,2, \ldots, \infty$, when we replace $H_{\mathcal{C}}$ by $e^{r} \circ H_{\mathcal{C}}(\mathrm{cf}(1.10))$ and $b \circ \tilde{f}$ by the $r$-fold (standard) suspension $E^{r}([b \circ \tilde{f}]) \in \pi_{m+r}\left(S^{n+r}\right)\left(\right.$ for $\left.\tilde{f}=\tilde{f}_{i}, a^{\circ} \circ \tilde{f}_{i}, i=1,2\right)$.

Case $3 \quad Y$ is simply connected and admits a fixed point free map a Deform $a$ to a base point preserving map $a^{\bullet}:\left(Y, y_{0}\right) \rightarrow\left(Y, y_{0}\right)$. Then

$N_{r}\left(f_{1}, f_{2}\right)$

$$
= \begin{cases}1 & \text { if } e^{r}\left(H_{\mathcal{C}}\left(f_{1}\right)\right) \neq e^{r}\left(H_{\mathcal{C}}\left(a^{\circ} \circ f_{2}\right)\right) \text { or } E^{r}\left(\left[b \circ f_{1}\right]\right) \neq E^{r}\left(\left[b \circ a \circ f_{2}\right]\right), \\ 0 & \text { otherwise. }\end{cases}
$$

In particular, the values which our Nielsen numbers may possibly assume are severely restricted. In fact, only two or at most three different values can occur:

Proposition 1.12 Let $0 \leq r \leq \infty, m \geq 2, Y$ and $k:=\# \pi_{1}(Y)$, as well as $\left[f_{1}\right],\left[f_{2}\right] \in$ $\pi_{m}(Y)$ be as in Theorem 1.11.

Then $N_{r}\left(f_{1}, f_{2}\right) \in\{0,1, k\}$. Furthermore, if $N_{r}\left(f_{1}, f_{2}\right) \notin\{0, k\}$, then the following restrictions must all be satisfied:

(i) $n$ is even and $m \geq n \geq 4$, or else $m=2$ and $Y=\mathbb{R P}(2)$.

(ii) The manifold $Y$ is closed, not orientable and not a product of two manifolds with strictly positive dimensions. $\pi_{1}(Y) \cong \mathbb{Z}_{2} \cdot \chi(Y) \neq 0 . Y$ admits no fixed point free selfmap. Also the homomorphism $i_{*}: \pi_{m}(Y \backslash\{*\}) \rightarrow \pi_{m}(Y)$ (induced by the inclusion of $Y$, punctured at some point $*$ ) is not surjective. Moreover, the composed homomorphism

$$
E \circ \partial_{Y}: \pi_{m}(Y) \stackrel{\partial_{Y}}{\longrightarrow} \pi_{m-1}\left(S^{n-1}\right) \stackrel{E}{\longrightarrow} \pi_{m}\left(S^{n}\right)
$$

is nontrivial; here $\partial_{Y}$ denotes the boundary homomorphism in the homotopy sequence of the tangent sphere bundle $S T(Y)$ over $Y$ and $E$ is the Freudenthal suspension.

A typical example of a manifold $Y$ that may satisfy all these restrictions is even dimensional real projective space $\mathbb{R P}(n)=S^{n} / \mathbb{Z}_{2} \cdot a$, the orbit space of the antipodal involution $a$. Section 4 below presents a detailed discussion of the more general case where $Y=S^{n} / G$ is an arbitrary spherical space form, ie, the orbit manifold of a smooth free action of a finite group $G$ on the $n$-sphere. Combining Theorem 1.11 with the results of our appendix we can express all Nielsen numbers $N_{r}\left(f_{1}, f_{2}\right)$ of 
maps $f_{1}, f_{2}: S^{m} \rightarrow S^{n} / G$ explicitly in terms of the more familiar Hopf invariants à la Hilton and James, and of the standard Freudenthal suspension. This allows many concrete computations. As a side result we note that all these Nielsen numbers do not depend on the $G$-action itself but only on the order \# $G$ of the group $G$. Furthermore, for many values of the codimension $m-n$ we have $N_{\infty}\left(f_{1}, f_{2}\right) \in\{0, \# G\}$.

We are particularly interested on those settings where the Nielsen numbers can possibly assume three distinct values (compare Proposition 1.12). Thus let $n \geq 2$ be even and $G \cong \mathbb{Z}_{2}$. In several important cases we are able to carry out our 2 -step program and compute all Nielsen numbers as well as the minimum number MCC.

Example 1.13 ( $m \leq 2 n-1, n \geq 2$ even) Given maps $f_{1}, f_{2}: S^{m} \rightarrow S^{n} / \mathbb{Z}_{2}$, all Nielsen numbers agree, $N_{r}\left(f_{1}, f_{2}\right)=N^{\#}\left(f_{1}, f_{2}\right)$ for $0 \leq r \leq \infty$, and are determined by Corollary 4.4(ii) or, equivalently, by Corollary 4.5 below.

If $m \leq 2 n-3$, then $\operatorname{MCC}\left(f_{1}, f_{2}\right)=N^{\#}\left(f_{1}, f_{2}\right)$.

If $m=2 n-2$, then $\operatorname{MCC}\left(f_{1}, f_{2}\right)=N^{\#}\left(f_{1}, f_{2}\right)$ except precisely if $n=16,32,64$ (or maybe 128), and $f_{1} \sim f_{2}=: f$, and $N^{\#}(f, f)=0$, but the lifting $\tilde{f}: S^{2 n-2} \rightarrow S^{n}$ of $f$ has a nontrivial Kervaire invariant $K(\tilde{f})=1$.

If $m=2 n-1$, then $\operatorname{MCC}\left(f_{1}, f_{2}\right)=N^{\#}\left(f_{1}, f_{2}\right)$ except precisely if $n \equiv 2(4), n \geq 6$, $f_{1} \sim f_{2}=: f, N^{\#}(f, f)=0$, and the Hopf invariant of the lifting $\tilde{f}: S^{2 n-1} \rightarrow S^{n}$ of $f$ is not divisible by 4 .

More details about this and the next example can be found in Section 5 below.

Example 1.14 ( $m \leq n+3, n \geq 2$ even) Given maps $f_{1}, f_{2}: S^{m} \rightarrow S^{n} / \mathbb{Z}_{2}$ and $0 \leq r \leq \infty$, we have

$$
\operatorname{MCC}\left(f_{1}, f_{2}\right)=N_{0}\left(f_{1}, f_{2}\right)=\cdots=N_{r}\left(f_{1}, f_{2}\right)=\cdots=N_{\infty}\left(f_{1}, f_{2}\right) .
$$

Ie, the Nielsen numbers do not depend on $r$ and agree with the minimum number MCC; they are determined by Corollary 4.4(ii) or, equivalently, by Corollary 4.5 below.

When the domain and target manifolds of $f_{1}, f_{2}$ have the same dimension $m=n$ (eg in fixed point theory) and also in Examples 1.13 and 1.14, our Nielsen numbers are independent of $r$. However, they can be quite distinct in general (and lose strength, but gain in computability as $r$ increases).

Given $Y, r, m$ and $X=S^{m}$ as in Theorem 1.11, a standard stability argument shows that $N_{r} \equiv N_{r+1} \equiv \cdots \equiv N_{\infty}$ for $r \geq m-2 n+2$; thus the number of possibly different Nielsen number functions $N_{r}$ is limited by the so-called "degree of instability" $m-2 n+3$ (cf $[16,1.12])$. This number is nearly attained in the following example. 
Example 1.15 $\left(m=16, Y=S^{6} / \mathbb{Z}_{2}\right)$ Here the Nielsen numbers $N_{r}$ determine five distinct functions on pairs $f_{1}, f_{2}: S^{16} \rightarrow S^{6} / \mathbb{Z}_{2}$ of maps:

$$
\mathrm{MC} \equiv \mathrm{MCC} \equiv N_{0} \not \equiv N_{1} \equiv N_{2} \not \equiv N_{3} \not \equiv N_{4} \not \equiv N_{5} \equiv N_{6} \equiv \cdots \equiv N_{\infty}
$$

(Stability arguments would not allow more than seven distinct such functions anyway.)

In Section 5 below we present criteria for compairing a Nielsen number $N_{r}$ to $N_{r+1}$. Then we discuss the dimension setting of Example 1.15 in detail, tabulating value distributions of the Nielsen numbers and determining the loose pairs $\left(\left[f_{1}\right],\left[f_{2}\right]\right) \in$ $\pi_{16}\left(S^{6} / G\right)^{2}, G=\mathbb{Z}_{2}$ or $\{0\}$.

In Section 6 below we finally come back to the central objects of study in topological coincidence theory: the minimum numbers MC and MCC of coincidence points and of coincidence path-components, resp. (cf (1.2), (1.3), and compare [3, page 9]). Given maps $f_{1}, f_{2}: S^{m} \rightarrow S^{n} / G$ we show that $\operatorname{MC}\left(f_{1}, f_{2}\right)=\operatorname{MCC}\left(f_{1}, f_{2}\right)$ whenever $\operatorname{MC}\left(f_{1}, f_{2}\right)$ is finite; moreover we give finiteness criteria for $\operatorname{MC}\left(f_{1}, f_{2}\right)$ in terms of Hopf-Hilton invariants.

Problem 1.16 Let $f_{1}, f_{2}: X \rightarrow Y$ be maps between arbitrary smooth connected manifolds, $X$ being compact.

Is $\operatorname{MC}\left(f_{1}, f_{2}\right)=\operatorname{MCC}\left(f_{1}, f_{2}\right)$ whenever $\operatorname{MC}\left(f_{1}, f_{2}\right)<\infty$ ? Also, give general complete criteria for $\operatorname{MC}\left(f_{1}, f_{2}\right)$ being finite.

Recall that the case $n=2$ may play a special role here (cf [11, Theorem 1.2(iii)]).

When the domain $X$ of the maps $f_{1}, f_{2}$ is a sphere $S^{m}$, a generic coincidence manifold can be contracted to a point within $S^{m}$. In the construction of the coincidence invariant $\omega_{r}\left(f_{1}, f_{2}\right)$ (cf (1.6)), this allows us to replace $E\left(f_{1}, f_{2}\right)$ by the loop space $\Omega Y$ of the target manifold $Y$; furthermore, $\bar{g}_{r}$ becomes a trivialisation of the normal bundle $v\left(i_{r}\right)$ (compare [10], [11] and also Section 2 below). Then the Pontryagin-Thom procedure transforms the bordism class $\left[i_{r}, \bar{g}, \tilde{g}\right]$ of geometric coincidence data (cf (1.6)) into an element of the homotopy group $\pi_{m+r}\left(S^{n+r} \wedge(\Omega Y)^{+}\right)$, where $(\Omega Y)^{+}$ denotes the loop space $\Omega Y$ with one disjointly added point. Moreover the transition from $\omega_{r}\left(f_{1}, f_{2}\right)$ to $\omega_{r+1}\left(f_{1}, f_{2}\right)$ corresponds to applying the standard Freudenthal suspension.

For all our calculations it is crucial to understand these groups and suspension homomorphisms in depth for $r=0,1, \ldots, \infty$. The necessary homotopy theoretical tools are provided in the appendix at the end of the paper where we interpret $S^{q} \wedge(\Omega Y)^{+}$ as the fiber of a Serre fibration for $q \geq 1$. 
Conventions and notation Throughout this paper $m, n \geq 1$, and $Y$ is a smooth connected $n$-dimensional manifold (Hausdorff, having a countable base) without boundary and with basepoint $y_{0}$. Let $\Omega Y=\Omega\left(Y, y_{0}\right)$ (and $(\Omega Y)^{+}$, resp.) denote the loop space (with a single disjoint point added, resp.). $E$ stands for the Freudenthal suspension. $T_{y}(Y)$ is the tangent space of $Y$ at a point $y \in Y$. If $p: \tilde{Y} \rightarrow Y$ denotes the universal covering we equip $\tilde{Y}$ with a basepoint $\tilde{y}_{0} \in p^{-1}\left(\left\{y_{0}\right\}\right)$. Identity maps are denoted by id. The symbols $\sim$ (or $\not$, resp.) mean freely homotopic (or not, resp.). $\# S$ is the cardinality of a set $S$.

\section{Acknowledgements}

It is a pleasure to thank Marek Golasinski for very valuable references.

This work was supported by DFG (Deutsche Forschungsgemeinschaft).

\section{The coincidence invariants $\omega_{r}$ and Hopf-Ganea homomor- phisms}

In this section we discuss our $\omega$-invariants and their homotopy theoretical interpretation - via the isomorphism $j_{*}$ (cf Proposition A.8 in the appendix below) - in terms of suspensions and Hopf-Ganea invariants.

Assume that $n \geq 2$. Fix a local orientation of the $n$-manifold $Y$ at its basepoint $y_{0}$ and an embedded path $\gamma$ in $Y$ from $y_{0}$ to some point $* \in Y, * \neq y_{0}$. (Constant maps with value $*$ will also be denoted by $*)$. Then, given $\left[f_{1}\right] \in \pi_{m}(Y, *)$ and $\left[f_{2}\right] \in \pi_{m}\left(Y, y_{0}\right)$, we can use the Pontryagin-Thom procedure to interpret $\omega^{\#}\left(f_{1}, f_{2}\right)=\omega_{0}\left(f_{1}, f_{2}\right)$ (cf (1.6)) as an element in $\pi_{m}\left(S^{n} \wedge(\Omega Y)^{+}\right)$(cf [11, Proposition 2.5]); more generally,

$$
\omega_{r}\left(f_{1}, f_{2}\right)=E^{r}\left(\omega^{\#}\left(f_{1}, f_{2}\right)\right) \in \pi_{m+r}\left(S^{n+r} \wedge(\Omega Y)^{+}\right), \quad r=0,1, \ldots, \infty,
$$

(compare (1.6) and (2.1') below) since the inclusion $\mathbb{R}^{m+i} \subset \mathbb{R}^{m+i+1}, i \geq 0$, induces the suspension homomorphism $E$. In addition

$$
\omega_{r}\left(f_{1}+f_{1}^{\prime}, f_{2}+f_{2}^{\prime}\right)=\omega_{r}\left(f_{1}, f_{2}\right)+\omega_{r}\left(f_{1}^{\prime}, f_{2}^{\prime}\right)
$$

for all $\left[f_{1}\right],\left[f_{1}^{\prime}\right] \in \pi_{m}(Y, *),\left[f_{2}\right],\left[f_{2}^{\prime}\right] \in \pi_{m}\left(Y, y_{0}\right)$ (cf $\left.[11,6.1]\right)$.

Let us describe these invariants more explicitly in the case when $f_{1} \equiv *$. Given $[f] \in \pi_{m}\left(Y, y_{0}\right)$, we may assume that $f: S^{m} \rightarrow Y$ is smooth with regular value $*$. Then $\omega^{\#}(*, f)$ is the nonstabilized bordism class of the triple $\left(C, \widetilde{g}, \bar{g}^{\#}\right)$ consisting of:

(i) The embedded smooth submanifold $C:=f^{-1}(\{*\})$ of $\mathbb{R}^{m} \subset \mathbb{R}^{m} \cup\{\infty\}=S^{m}$. 
(ii) The map $\tilde{g}$ from $C$ to the loop space $\Omega Y$, defined as follows: pick a homotopy $G: C \times I \rightarrow S^{m}$ from the inclusion $C \subset S^{m}$ to the constant map with value $\infty$ (= the basepoint of $\left.S^{m}\right)$. Then $\tilde{g}(x)$ is the (concatenated) loop $\gamma \cdot f(G(x,-))$, $x \in C$.

(iii) $(-1) \cdot \bar{g}^{\#}$ is the isomorphism from the normal bundle of $C$ in $\mathbb{R}^{m}$ to the trivial bundle (over $C$ ) with fiber $T_{*}(Y) \cong T_{y_{0}}(Y) \cong \mathbb{R}^{n}$, induced by the tangent map of $f$ and the chosen path $\gamma$.

We use the Pontryagin-Thom procedure to identify the group of nonstabilized bordism classes of such triples with the homotopy group $\pi_{m}\left(S^{n} \wedge\left((\Omega Y)^{+}\right)\right.$) (for more details, see [11]).

If we forget about embeddings and consider $C$ only as an abstract $(m-n)$-dimensional manifold, equipped with the map $\tilde{g}$ and with the stable framing determined by $\bar{g}^{\#}$, we obtain the framed bordism class

$$
\omega_{\infty}(*, f)=\left[C, \tilde{g}, \bar{g}_{\infty}\right] \in \Omega_{m-n}^{f r}(\Omega Y)=\lim _{r \rightarrow \infty} \pi_{m+1}\left(S^{n+r} \wedge(\Omega Y)^{+}\right),
$$

which was discussed in detail in [10].

Now let $B \subset Y$ be a smoothly embedded compact $n$-ball with center point $*$ such that $y_{0}$ lies in the boundary sphere $\partial B$ and $B$ contains the image of $\gamma$. We obtain a pinching map

$$
\text { pinch: } Y=(Y-\stackrel{\circ}{B}) \cup_{\partial B} B \rightarrow Y / \partial B \cong S^{n} \vee Y \text {, }
$$

which collapses $\partial B$ to a point.

Theorem 2.3 If $Y$ is a simply connected, oriented $n$-dimensional manifold, $n \geq 2$, the diagram of homomorphisms

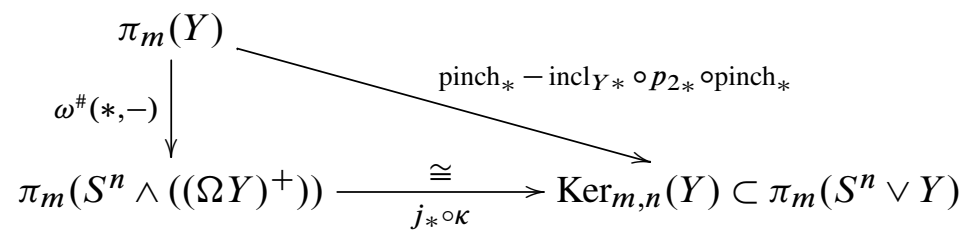

commutes. (Here $j_{*}$ is as defined in the appendix below, cf (A.7) and Proposition A.8; moreover $\kappa$ denotes the involution induced by $(-1)$ times the identity map on $\mathbb{R}^{n} \subset$ $S^{n}=\mathbb{R}^{n} \cup\{\infty\}$.) 
Proof The corresponding result for general $Y$ (with arbitrary finite fundamental group) was established in [11, Theorem 7.2]. Here we give a different geometric proof for the special case $Y=S^{n}$ (which is relevant for spherical space forms).

Given $[f] \in \pi_{m}\left(S^{n}\right)$, we may assume that $f$ has the following standard form: there exists a smoothly embedded tubular neighbourhood

$$
T=C \times B^{n} \subset \mathbb{R}^{m} \subset S^{m}
$$

such that $f \mid T$ is the projection

$$
C \times B^{n} \rightarrow B^{n} / \partial B^{n}=S^{n}=\mathbb{R}^{n} \cup\{\infty\}
$$

and $f(x)=y_{0}=\infty$ for all $x \notin T$. In the spirit of Pontryagin-Thom we may interpret $\operatorname{pinch}_{*}([f])$ by the framed link $C \amalg C^{\prime} \subset \mathbb{R}^{m}$ consisting of the (neighbouring "parallel") components $C=C \times\{0\}=f^{-1}(\{*\})$ and $C^{\prime}=C \times\left\{z_{0}\right\}$ for some $z_{0} \in \stackrel{\circ}{B}^{n} \backslash\{0\}$. Then $\operatorname{incl}_{Y *} \circ p_{2} \circ \operatorname{pinch}_{*}([f])$ corresponds to the translated framed submanifold $C^{\prime \prime}=C^{\prime}+v_{0} \subset \mathbb{R}^{m}$, pushed away by some big vector $v_{0} \in \mathbb{R}^{m}$, so that it does not link with $C \times B^{n}$ anymore.

Consider the homotopy

$$
G^{\prime}: C^{\prime} \times I \rightarrow \mathbb{R}^{m}, \quad G^{\prime}(x, t):=x+t v_{0},
$$

and the embedding

$$
E: C^{\prime} \times I \subset \mathbb{R}^{m} \times I, \quad E(x, t):=\left(x+t v_{0}, t\right),
$$

$(x, t) \in C^{\prime} \times I$. We may assume that $* \in S^{n}$ is a regular value of $f \circ G^{\prime}$ so that $E\left(C^{\prime} \times I\right)$ intersects $C \times I$ transversely in an embedded submanifold

$$
K \subset C \times(0,1) \subset \mathbb{R}^{m} \times(0,1) .
$$

Pick $\delta>0$ such that the $\delta$-neighbourhood of $K$ is still embedded, and so is the $\delta \cdot t-$ neighbourhood of $(c, t) \in K$, growing as $t>0$ increases from one intersection of $E$ with $\{c\} \times I$ to a higher one, for any $c \in C$. Now remove the $\delta \cdot t-$ ball $B^{\prime} \subset E\left(C^{\prime} \times I\right)$ around each point $(c, t) \in K$ and replace it by the cylinder $\partial B^{\prime} \times[t, 1] \subset \mathbb{R}^{m} \times I$.

After smoothing corners we obtain an embedded framed bordism in $\left(\mathbb{R}^{m} \backslash C\right) \times I$ from $C^{\prime} \times\{0\}$ to the disjoint union of $C^{\prime} \times\{1\}$ with a framed submanifold $\widehat{C} \subset$ $\mathbb{R}^{m} \times\{1\}$ that lies in the $\delta$-neighbourhood of $C \times\{1\}$ (see Figure 1). But the link $C \amalg \widehat{C} \subset \mathbb{R}^{m}$ represents $j_{*} \circ \kappa\left(\omega^{\#}(*, f)\right)$. This follows from Proposition A.11 below if we construct $\omega^{\#}(*, f)$ using the homotopy $G^{\prime}$ (cf (2.4)) as well as the straight path between 0 and $z_{0}$ in $B^{n}$ and a local isotopy along this path. Therefore $\operatorname{pinch}_{*}([f])=$ $\operatorname{incl}_{Y *} \circ p_{2 *} \circ \operatorname{pinch}_{*}([f])+j_{*} \circ \kappa\left(\omega^{\#}(*, f)\right)$. 


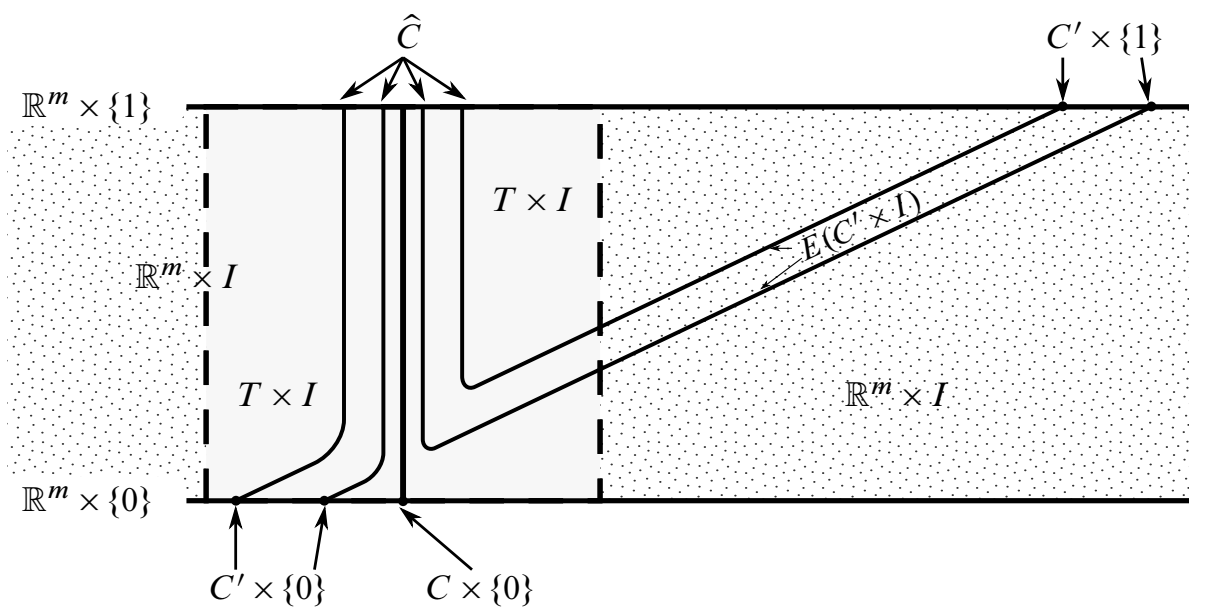

Figure 1: The bordism that proves Theorem 2.3 in the case $Y=S^{n}$

For all $m, q \geq 1$ there exists a canonical decomposition

$$
\operatorname{Ker}_{m, q}(Y)=\pi_{m}\left(S^{q}\right) \oplus \pi_{m}\left(S^{q} b Y\right),
$$

where $S^{q}$ b $Y$ denotes the homotopy fiber of the inclusion $S^{q} \vee Y \subset S^{q} \times Y$ (cf [7, (9); $6,6.7])$.

Corollary 2.6 Let $B$ be a compact $n$-ball embedded in the 1-connected oriented manifold $Y$ (as in Theorem 2.3). For all $[f] \in \pi_{m}(Y)$,

$$
j_{*} \circ \kappa\left(\omega^{\#}(*, f)\right)=\left([b \circ f], H_{\mathcal{C}}(f)\right) \in \pi_{m}\left(S^{n}\right) \oplus \pi_{m}\left(S^{n} b Y\right),
$$

where $b$ and $H_{\mathcal{C}}$ are defined as a (1.8) and (1.9).

Thus our basic coincidence invariant $\omega^{\#}(*, f)$ turns out to be an enriched Hopf-Ganea invariant. For a proof and further details, see [11, (63)-(65)].

Corollary 2.7 For all $r=0,1, \ldots, \infty$ and $[f] \in \pi_{m}(Y)$ we have $j_{*}\left(E^{r}\left(\kappa\left(\omega^{\#}(*, f)\right)\right)\right)=\left(E^{r}([b \circ f]), e^{r}\left(H_{\mathcal{C}}(f)\right)\right) \in \pi_{m+r}\left(S^{n+r}\right) \oplus \pi_{m+r}\left(S^{\left.n+r_{b} Y\right)}\right.$. In particular, $\omega_{r}(*, f)=0$ if and only if $E^{r}([b \circ f])=0$ and $e^{r}\left(H_{\mathcal{C}}(f)\right)=0$.

Proof This follows from the fact that $j_{*}$ is injective and compatible with suspensions (cf Theorem A.15(a) below). Moreover, $\omega_{r}=E^{r} \circ \omega^{\#}(\mathrm{cf}(2.1))$ agrees with $E^{r} \circ \kappa \circ \omega^{\#}$ up to an involution on $\pi_{*}\left(S^{n+r} \wedge(\Omega Y)^{+}\right)$of the form $(d \wedge \text { id })_{*}$, where the map $d: S^{n+r} \rightarrow S^{n+r}$ has degree $(-1)^{n}$. 


\section{Computing Nielsen numbers}

In this section we prove Theorem 1.11 and Proposition 1.12

Let $p: \tilde{Y} \rightarrow Y$ be a universal covering map of the $n$-dimensional manifold $Y, n \geq 2$, and pick a basepoint $\tilde{y}_{0} \in \tilde{Y}$ such that $p\left(\tilde{y}_{0}\right)=y_{0}$. Also denote the number of path-components of the loop space $\Omega Y=\Omega\left(Y, y_{0}\right)$ by

$$
k:=\# \pi_{1}(Y)=\# \pi_{0}(\Omega Y), \quad 1 \leq k \leq \infty .
$$

Given homotopy classes $[f],\left[f_{1}\right],\left[f_{2}\right], \ldots \in \pi_{m}\left(Y, y_{0}\right), m \geq 2$, let $[\tilde{f}],\left[\tilde{f}_{1}\right],\left[\tilde{f}_{2}\right], \ldots \in$ $\pi_{m}\left(\tilde{Y}, \tilde{y}_{0}\right)$ be their liftings.

Recall that the Nielsen number $N_{r}\left(f_{1}, f_{2}\right)$ is extracted from the coincidence data $\left(i_{r}, \tilde{g}, \bar{g}_{r}\right)$ of a generic coincidence manifold $C$ (cf (1.1)) as follows, $r=0,1, \ldots, \infty$. Since the domain of $f_{1}, f_{2}$ is a sphere, $\tilde{g}$ maps $C$ into the loop space $\Omega Y$ (after suitable homotopies; cf [10, 2.4], and compare also Section 1 above). Thus $C$ is the disjoint union of the Nielsen classes $C_{A}=\widetilde{g}^{-1}(A), A \in \pi_{0}(\Omega Y)$. Such a Nielsen class $C_{A}$ is called nonessential or essential, according to whether or not the coincidence data $\left(i_{r}, \tilde{g}, \bar{g}_{r}\right)$, when restricted to $C_{A}$, form a nullbordant triple. By definition $N_{r}\left(f_{1}, f_{2}\right) \in$ $\{0,1, \ldots, k\}$ is the number of essential Nielsen classes.

Clearly Nielsen numbers do not depend on the choice of the local orientation of $Y$ at $y_{0}$ and of the path $\gamma$, which play a role in the construction of $\omega_{r}\left(f_{1}, f_{2}\right)$ (compare the proof of Corollary 2.6).

Proof of Theorem 1.11 In Case 1 of the theorem, our claim follows from [12, Proposition 1.3].

If $k$ is finite and $n \geq 2$, consider first the coincidence data of a pair of the form $(*, f)$ where, $* \neq y_{0}$ and $[f] \in \pi_{m}\left(Y, y_{0}\right)$. After suitable isotopies of $\tilde{Y}$ and deformations of $\tilde{f}$ we may assume that:

(i) There is a smoothly embedded open $n$-ball $\stackrel{\circ}{B} \subset \tilde{Y} \backslash\left\{\tilde{y}_{0}\right\}$ that contains all the points $p^{-1}(\{*\})=\left\{\widetilde{*}=\widetilde{*}_{1}, \widetilde{*}_{2}, \ldots, \widetilde{*}_{k}\right\} \subset \widetilde{Y}$ over $* \in Y$.

(ii) $\tilde{f}$ is smooth with regular value $\widetilde{*}=\widetilde{*}_{1}$ and maps a tubular neighbourhood

$$
\widetilde{C} \times \stackrel{\circ}{B}=\stackrel{\circ}{T} \subset \mathbb{R}^{m} \subset \mathbb{R}^{m} \cup\{\infty\}=S^{m}
$$

of $\widetilde{C}:=\widetilde{f}^{-1}(\{\widetilde{*}\})$ to $\stackrel{\circ}{B}$ via the obvious projection.

(iii) $\tilde{f}\left(S^{m} \backslash \stackrel{\circ}{T}\right) \subset \tilde{Y} \backslash \stackrel{\circ}{B}$. 
Then the generic coincidence manifold $C:=f^{-1}(\{*\})=\tilde{f}^{-1}\left(\left\{\tilde{*}_{1}, \widetilde{*}_{2}, \ldots, \widetilde{*}_{k}\right\}\right)$ consists of the ("parallel") Nielsen classes

$$
\widetilde{C}_{i}=\widetilde{C} \times\left\{\widetilde{*}_{i}\right\} \subset \widetilde{C} \times \stackrel{\circ}{B} \subset S^{m}, \quad i=1, \ldots, k,
$$

which are simultaneously either all nonessential or essential, according to whether the coincidence data of $\widetilde{C}=\widetilde{C} \times\left\{*_{1}\right\}$ are nullbordant (or, equivalently $\omega_{r}(\widetilde{*}, \tilde{f})=0$ ) or not. Indeed, given a homotopy $G: \widetilde{C} \times I \rightarrow S^{m}$ from the inclusion $\widetilde{C} \subset S^{m}$ to a constant map at $\infty$, base the construction of the $\omega$-invariant on the concatenation of $G$ with the straight path $\widetilde{c}_{i}$ from $\left\{\widetilde{*}_{i}\right\}$ to $\{\widetilde{*}\}$ in $\stackrel{\circ}{B}, i=1, \ldots, k$ (see the beginning of our Section 2 above). Since the loops $p \circ \widetilde{c}_{i}$ in $Y$ are pairwise nonhomotopic we get the Nielsen decomposition $C=\amalg \widetilde{C}_{i}$ with equally strong components.

In contrast, the coincidence data $\left[i_{r}, \tilde{g}, \bar{g}_{r}\right]$ of a pair of the form $(f, f)$ have the special property that $\widetilde{g}$ is homotopic to a constant map ( $\mathrm{cf}[11,(21)])$. Thus the $\omega$-invariants of $(f, f)$ and $(\tilde{f}, \tilde{f})$ are equally strong and nontrivial precisely if the path-component of the trivial loop in $\Omega Y$ corresponds to an essential Nielsen class.

Next consider an arbitrary pair $\left(f_{1}, f_{2}\right),\left[f_{1}\right],\left[f_{2}\right] \in \pi_{m}\left(Y, y_{0}\right)$. Use the chosen path $\gamma$ from $y_{0}$ to $*$ (cf Section 2) and a small neighbourhood of the basepoint $\infty$ in $S^{m}$ to deform $f_{1}$ to a map $f_{1}^{\prime}:\left(S^{m}, \infty\right) \rightarrow(Y, *)$. According to $(2.2)$,

$$
\left(\omega_{r}\left(f_{1}, f_{2}\right)=\right) \omega_{r}\left(f_{1}^{\prime}, f_{2}\right)=\omega_{r}\left(f_{1}^{\prime}, f_{1}\right)-\omega_{r}\left(*, f_{1}\right)+\omega_{r}\left(*, f_{2}\right) .
$$

Applying our previous discussion to $[f]=\left[f_{1}\right]-\left[f_{2}\right]$, we see that the nontrivial elements of $\pi_{0}(\Omega Y) \cong \pi_{1}(Y)$ yield essential Nielsen classes if and only if

$$
\omega_{r}\left(\widetilde{*}, \widetilde{f}_{1}\right) \neq \omega_{r}\left(\widetilde{*}, \tilde{f}_{2}\right) .
$$

The trivial element of $\pi_{0}(\Omega Y)$ contributes an essential Nielsen class precisely if

$$
\omega_{r}\left(\tilde{f}_{1}^{\prime}, \tilde{f}_{1}\right)-\omega_{r}\left(\widetilde{*}, \tilde{f}_{1}\right)+\omega_{r}\left(\widetilde{*}, \tilde{f}_{2}\right) \neq 0 .
$$

Now assume that $\tilde{Y}$ allows a fixed point free selfmap $a$. It is freely homotopic to a basepoint preserving map

$$
a:\left(\tilde{Y}, \tilde{y}_{0}\right) \rightarrow\left(\tilde{Y}, \tilde{y}_{0}\right) .
$$

Then $\omega_{r}\left(\tilde{f_{1}^{\prime}}, a \cdot \widetilde{f_{1}}\right)=\omega_{r}\left(\tilde{f_{1}}, a \circ \tilde{f_{1}}\right)=0$ and

$$
\omega_{r}\left(\tilde{f_{1}^{\prime}}, \tilde{f_{1}}\right)=\omega_{r}\left(\tilde{f_{1}^{\prime}}, \tilde{f_{1}}\right)-\omega_{r}\left(\tilde{f_{1}^{\prime}}, a \circ \tilde{f}_{1}\right)=\omega_{r}\left(\widetilde{*}, \tilde{f}_{1}\right)-\omega_{r}\left(\widetilde{*}, a \circ \tilde{f}_{1}\right) .
$$

Therefore condition (3.2) takes the form

$$
\omega_{r}\left(\tilde{*}, a \circ \tilde{f}_{1}\right) \neq \omega_{r}\left(\widetilde{*}, \tilde{f}_{2}\right) .
$$


This agrees with condition (3.1) if the Euler characteristic of $\tilde{Y}$ vanishes (eg when $n$ is odd); indeed, a vector field without zeros yields a fixed point free selfmap $a$ of $\tilde{Y}$ that is isotopic to the identity map $a^{*}=\mathrm{id}$.

Finally apply the isomorphism $j_{*}$ to the conditions (3.1) and (3.2.a) and use Corollaries 2.6 and 2.7. Also note that $H_{\mathcal{C}}\left(\tilde{f}_{1}\right)=H_{\mathcal{C}}\left(a^{\circ} \circ \tilde{f}_{1}\right)$ since $\omega_{r}\left(\tilde{f}_{1}^{\prime}, \tilde{f}_{1}\right)$, and $j_{*}\left(\omega_{r}\left(\tilde{f}_{1}^{\prime}, \tilde{f}_{1}\right)\right)$, resp., lie already in the subgroup $\pi_{m}\left(S^{n}\right)$ of $\pi_{m}\left(S^{n} \wedge(\Omega \tilde{Y})^{+}\right)$, and of $\operatorname{Ker}_{m, n}(\tilde{Y})$, resp. (compare $(2.5)$ and $\left.[13,5.6]\right)$. This completes the proof of Theorem 1.11.

Proof of Proposition 1.12 We need to study only the arguments in the previous proof that deal with Case 2 of Theorem 1.11. All Nielsen classes are simultaneously essential or nonessential (ie, $\left.N_{r}\left(f_{1}, f_{2}\right) \in\{0, k\}\right)$ except possibly when $\omega_{r}\left(\tilde{f}_{1}, \tilde{f}_{1}\right) \neq 0$ (cf (3.2)). But in this case also $\omega_{r}\left(f_{1}, f_{1}\right)=E^{r}\left(\omega^{\#}\left(f_{1}, f_{1}\right)\right)$ and hence $\omega^{\#}\left(f_{1}, f_{1}\right)$ are nontrivial. Thus all the restrictions listed in Proposition 1.12 follow from [12, Proposition 1.3; 14, Theorem 1.32].

\section{Spherical space forms}

In this section we illustrate Theorem 1.11 with examples where $Y$ is an arbitrary spherical space form $S^{n} / G$. Here our criteria can be expressed in terms of the Hopf-Hilton invariant homomorphisms

$$
h_{j}^{\prime}: \pi_{m}\left(S^{n}\right) \rightarrow \pi_{m}\left(S^{n+j(n-1)}\right), \quad j=1,2, \ldots,
$$

which correspond to the basic Whitehead products

$$
w_{j}^{\prime}:=\left[\iota_{2}, \ldots,\left[\iota_{2},\left[\iota_{1}, \iota_{2}\right] \cdots\right]\right] \in \pi_{n+j(n-1)}\left(S^{n} \vee S^{n}\right)
$$

with one factor $\iota_{1}$ and $j$ factors $\iota_{2}$ (cf Hilton [8]). Define

$$
h^{\prime}:=\left(h_{1}^{\prime}, h_{2}^{\prime}, \ldots\right): \pi_{m}\left(S^{n}\right) \rightarrow \bigoplus_{j \geq 1} \pi_{m}\left(S^{n+j(n-1)}\right)
$$

and $h:=\left(\mathrm{id}, h^{\prime}\right)$. Thus, eg,

$$
E^{r} \circ h:=\left(E^{r}, E^{r} \circ h_{1}^{\prime}, E^{r} \circ h_{2}^{\prime}, \ldots\right) .
$$

Theorem 4.3 Let $G$ be a finite group acting smoothly and freely on the sphere $S^{n}$ and let $Y=S^{n} / G$ be the resulting orbit space. Assume $m, n \geq 2$ and $0 \leq r \leq \infty$. Also let $\iota \in \pi_{n}\left(S^{n}\right)$ be represented by the identity map id.

For all homotopy classes $\left[f_{i}\right] \in \pi_{m}\left(S^{n} / G, y_{0}\right)$ and their liftings $\left[\tilde{f}_{i}\right] \in \pi_{m}\left(S^{n}, \tilde{y}_{0}\right)$, $i=1,2$, we have: 
If $n$ is odd, then

$$
N_{r}\left(f_{1}, f_{2}\right)= \begin{cases}\# G & \text { if } E^{r} \circ h\left(\left[\tilde{f}_{1}\right]\right) \neq E^{r} \circ h\left(\left[\tilde{f}_{2}\right]\right), \\ 0 & \text { otherwise. }\end{cases}
$$

If $n$ is even and $G=0$, then

$$
N_{r}\left(f_{1}, f_{2}\right)= \begin{cases}1 & \text { if } E^{r} \circ h\left(\left[\tilde{f}_{1}\right]\right) \neq E^{r} \circ h\left((-\iota) \circ\left[\tilde{f}_{2}\right]\right) ; \\ 0 & \text { otherwise. }\end{cases}
$$

If $n$ is even and $G \cong \mathbb{Z}_{2}$, then

$N_{r}\left(f_{1}, f_{2}\right)$

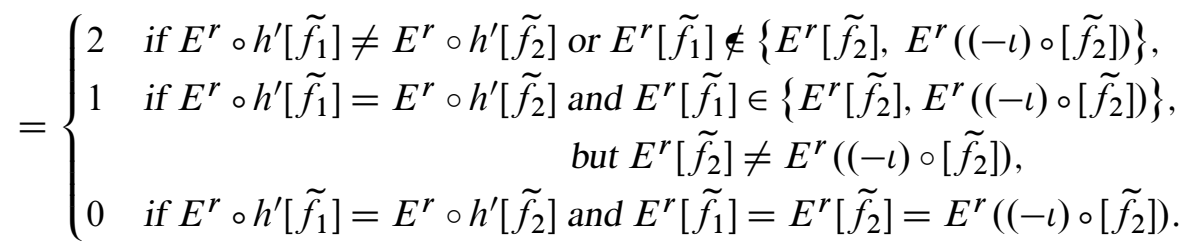

In particular, all these Nielsen numbers depend only on the order \# $G$ of the group $G$ and not on the $G$-action itself.

Note that $\# G \leq 2$ when $n$ is even (as seen by a simple argument involving Euler characteristics).

Corollary 4.4 (Case $r=0)$ Recall that $N_{0} \equiv N^{\#}(\operatorname{cf}(1.5))$. Let $a: S^{n} \rightarrow S^{n}$ denote the antipodal map.

(i) Suppose that $n$ is odd or $G=0$. Then

$$
N_{0}\left(f_{1}, f_{2}\right)= \begin{cases}\# G & \text { if } \tilde{f}_{1} \not a \circ \tilde{f}_{2}, \\ 0 & \text { if } \tilde{f}_{1} \sim a \circ \widetilde{f}_{2} .\end{cases}
$$

In particular, if $n \geq 3$ is odd then $N_{0}\left(f_{1}, f_{2}\right)$ takes the value 0 or \#G according to whether $\widetilde{f}_{1}$ is (freely) homotopic to $\widetilde{f}_{2}$ or not, respectively.

(ii) Suppose that $n$ is even and $G \cong \mathbb{Z}_{2}$. Then

$$
N_{0}\left(f_{1}, f_{2}\right)=\left\{\begin{array}{l}
2 \text { if } \tilde{f}_{1} \nsim \tilde{f}_{2} \text { and } \tilde{f}_{1} \nsim a \circ \tilde{f}_{2}, \\
1 \quad \text { if } \tilde{f}_{1} \sim \tilde{f}_{2} \text { or } \tilde{f}_{1} \sim a \circ \tilde{f}_{2}, \text { but } \tilde{f}_{2} \nsucc a \circ \tilde{f}_{2}, \\
0 \quad \text { if } \tilde{f}_{1} \sim \tilde{f}_{2} \sim a \circ \tilde{f}_{2} .
\end{array}\right.
$$


Corollary 4.5 (Case $r \geq 1)$ Here $E^{r}\left((-\iota) \circ\left[\tilde{f}_{2}\right]\right)=-E^{r}\left(\left[\tilde{f}_{2}\right]\right)$. We get, eg, for $n$ even, $G \cong \mathbb{Z}_{2}$ :

$N_{r}\left(f_{1}, f_{2}\right)$

$=\left\{\begin{array}{l}2 \quad \text { if } E^{r} \circ h^{\prime}\left(\tilde{f}_{1}\right) \neq E^{r} \circ h^{\prime}\left(\tilde{f}_{2}\right) \text { or } E^{r}\left(\tilde{f}_{1}\right) \neq \pm E^{r}\left(\tilde{f}_{2}\right), \\ 1 \quad \text { if } E^{r} \circ h^{\prime}\left(\tilde{f}_{1}\right)=E^{r} \circ h^{\prime}\left(\tilde{f}_{2}\right) \text { and } E^{r}\left(\tilde{f}_{1}\right)= \pm E^{r}\left(\tilde{f}_{2}\right) \text { has order more than } 2, \\ 0 \quad \text { if } E^{r} \circ h^{\prime}\left(\tilde{f}_{1}\right)=E^{r} \circ h^{\prime}\left(\tilde{f}_{2}\right) \text { and } E^{r}\left(\tilde{f}_{1}\right)= \pm E^{r}\left(\tilde{f}_{2}\right) \text { has order at most } 2 .\end{array}\right.$

Note that Hopf invariants play no role here in our criteria for the basic Nielsen number $N^{\#}=N_{0}$. However they are often decisive when $r \geq 1$.

Example 4.6 $(m=3, n=2$ and $G=0)$ For all maps $f_{1}, f_{2}: S^{3} \rightarrow S^{2}$ and $0 \leq r \leq \infty$ we have: $N_{r}\left(f_{1}, f_{2}\right)$ equals 0 or 1 , resp., according to whether $f_{1} \sim f_{2}$ or $f_{1} \not f_{2}$, resp. When $r \geq 1$, this is detected only by $E^{r} h^{\prime}=E^{r} h_{1}^{\prime}$ or, equivalently, by the classical Hopf invariant $H$. Indeed,

$$
h^{\prime}\left(f_{i}\right)= \pm H\left(f_{i}\right) \circ \iota \in \pi_{3}\left(S^{3}\right) \cong \mathbb{Z}, \quad i=1,2
$$

(cf Whitehead [18, XI, 8.17]) persists under all iterated suspensions; in contrast, $E^{r}\left(f_{i}\right)$ measures only values modulo 2 in $\pi_{r+3}\left(S^{r+2}\right) \cong \mathbb{Z}_{2}$ when $r \geq 1$.

Corollary 4.7 (Case $r=\infty$ ) For all $\left[f_{1}\right],\left[f_{2}\right] \in \pi_{m}\left(S^{n}\right), m, n \geq 2$, we have $N_{\infty}\left(f_{1}, f_{2}\right)= \begin{cases}0 & \text { if } E^{\infty} \circ \gamma_{j}\left(\left[f_{1}\right]\right)=E^{\infty} \circ \gamma_{j}\left(\left((-1)^{n+1} \cdot \iota\right) \circ\left[f_{2}\right]\right) \text { for all } j \geq 1, \\ 1 & \text { otherwise. }\end{cases}$ Here $E^{\infty} \circ \gamma_{j}: \pi_{m}\left(S^{n}\right) \rightarrow \pi_{m-1-j(n-1)}^{S}$ is defined by the infinitely suspended HopfJames invariant, $j=1,2, \ldots$.

This was proved already in [10, Section 8] by interpreting $E^{\infty} \circ \gamma_{j}$ via $(j-1)$-tuple self-intersections of framed immersions.

Corollary 4.8 Let $Y=S^{n} / G$ and $m, n \geq 2$ be as in Theorem 4.3. Assume that $2 \alpha=0$ for all $\alpha \in \pi_{m-n}^{S}$ (according to the tables in Toda [17] this holds eg when $m-n=$ $1,2,4,5,6,8,9,12,14,16$ or 17$)$. Then $N_{\infty}\left(f_{1}, f_{2}\right) \in\{0, \# G\}$ for all $\left[f_{1}\right],\left[f_{2}\right] \in$ $\pi_{m}\left(S^{n} / G\right)$.

Hopf-James invariants occur not only in the criteria which determine Nielsen numbers (as eg in Corollary 4.7). They play also an important role - via EHP-sequences - in the computations needed to decide whether these criteria are fulfilled. 
Proof of Theorem 4.3 and Corollaries 4.4, 4.5 and 4.7 We adopt the arguments in the proof of Theorem 1.11; thus $\tilde{Y}=S^{n}$. In view of the criteria (3.1) and (3.2.a), we need to apply Theorem 2.3 only to (lifted) homotopy classes $\tilde{\varphi} \in \pi_{m}\left(S^{n}\right)$. For the calculation of Nielsen numbers, we may assume that the $n$-ball $B \subset S^{n}$ (used in (1.8), (1.9) and in the construction of the pinching map in Theorem 2.3) is a suitable half-sphere, endowed with the standard orientation of $S^{n}$. Then $b \sim$ id in (1.8) and

$$
\operatorname{pinch}_{*}(\tilde{\varphi})=\left(\iota_{1}+\iota_{2}\right) \circ \tilde{\varphi} \in \pi_{m}\left(S^{n} \vee S^{n}\right),
$$

where $\iota_{1}$ and $\iota_{2}$ are represented by the two obvious inclusions of $S^{n}$ onto $S^{n} \vee S^{n}$. Using Hilton's choice of basic Whitehead products and applying his [8, Theorem A], we conclude that

(4.10) $\left(\operatorname{pinch}_{*}-\iota_{2 *} \circ p_{2 *} \circ \operatorname{pinch}_{*}\right)(\widetilde{\varphi})=\iota_{1} \circ \widetilde{\varphi}+\sum_{j \geq 1} w_{j}^{\prime} \circ h_{j}^{\prime}(\widetilde{\varphi})+\sum w_{k}^{\prime \prime} \circ h_{k}^{\prime \prime}(\widetilde{\varphi})$;

here the last two sums to the right involve those basic Whitehead products of $\iota_{1}$ and $\iota_{2}$ that contain $\iota_{1}$ precisely once (cf (4.2)), and at least twice, respectively.

Now according to Theorem A.15(a) below and Theorem 2.3,

$$
\omega_{r}(\widetilde{*}, \tilde{\varphi})=E^{r}\left(\omega^{\#}(\widetilde{*}, \tilde{\varphi})\right)=0
$$

or, equivalently, $E^{r}\left(\kappa\left(\omega^{\#}(\widetilde{*}, \widetilde{\varphi})\right)\right)=0$ (cf Theorem 2.3$)$ if and only if the iterated partial suspension homomorphism $e^{r}$ annihilates the right hand term in Equation (4.10). Denote this term by $\tau$. It vanishes precisely if its first summand and hence $\tilde{\varphi}$ itself does (by [8, Theorem A]). When $r \geq 1$ Theorem A.15(b) and Corollary A.16 in our appendix, together again with Hilton's result (applied to $S^{n+r} \vee S^{n}$ ), imply that

$$
e^{r}(\tau)=\iota_{1} \circ E^{r}(\tilde{\varphi})+\sum_{j \geq 1} e^{r}\left(w_{j}^{\prime}\right) \circ E^{r}\left(h_{j}^{\prime}(\tilde{\varphi})\right)=0
$$

if and only if $E^{r}(\widetilde{\varphi})=0$ and $\pm E^{r} \circ h_{j}^{\prime}(\widetilde{\varphi})=0$ for all $j \geq 1$, ie, $E^{r} \circ h(\widetilde{\varphi})=0$.

Finally, put $\tilde{\varphi}:=\left[\tilde{f}_{1}\right]-( \pm \iota) \circ\left[\tilde{f}_{2}\right]$ and apply our criteria (3.1) and (3.2.a); note also that the antipodal map $a$ is freely homotopic to a representative $a^{\cdot}$ of the generator $(-1)^{n+1} \cdot \iota$ of $\pi_{n}\left(S^{n}, y_{0}\right)$. Theorem 4.3 and its corollaries, 4.4 and 4.5 , follow. For Corollary 4.7 compare also Brown and Schirmer [4, Theorem 4.18].

Remark 4.11 The discussion following formula (3.3) implies, in particular, that

$$
h^{\prime}([f])=h^{\prime}\left(\left((-1)^{n+1} \cdot \iota\right) \circ[f]\right)
$$

for all $[f] \in \pi_{m}\left(S^{n}\right), m, n \geq 2$. This has been used to simplify the criterion in Example 4.6. 
Proof of Corollary 4.8 As in the proof of Proposition 1.12 we need to show only that $\omega_{\infty}\left(\widetilde{f}_{1}, \widetilde{f}_{1}\right)$ vanishes (compare (3.2)) or, in the language of [10, Theorem 1.14], that

$$
\widetilde{\omega}_{j}\left(\tilde{f}_{1}, \tilde{f}_{1}\right):=h_{j}\left(\widetilde{\omega}\left(\tilde{f}_{1}, \tilde{f}_{1}\right)\right)=0, \quad j=1,2, \ldots
$$

When $j \geq 2$, the "Hopf invariant component" $h_{j}\left(\widetilde{\omega}\left(\tilde{f}_{1}, \tilde{f}_{1}\right)\right)$ is indeed trivial in this selfcoincidence situation (since $\widetilde{\omega}\left(\tilde{f}_{1}, \tilde{f}_{1}\right)$ lies already in the subgroup $\Omega_{m-n}^{f r}$ of $\Omega_{m-n}^{f r}(\Omega Y)$. Similarly

$$
\widetilde{\omega}_{1}\left(\widetilde{f}_{1}, \widetilde{f}_{1}\right)=\widetilde{d e g} g_{1}\left(\tilde{f}_{1}\right) \pm \widetilde{d e g}{ }_{1}\left(\tilde{f}_{1}\right) \in \pi_{m-n}^{S}
$$

(cf [10, Theorem 1.14]) vanishes in view of our assumption $2 \cdot \pi_{m-n}^{S}=0$.

Note that this assumption cannot be dropped. Eg, if $n=4,8,12,14,16$ or 20 , then there exist infinitely many homotopy classes $[f] \in \pi_{2 n-1}(\mathbb{R P}(n))$ such that $\omega_{\infty}(f, f) \neq 0$ or, equivalently, $N_{\infty}(f, f)=1$. Indeed, apply Corollary 4.5 to desuspensions of elements of order greater than 2 in $\pi_{n-1}^{S}$ (see also [12, Example 1.26]).

\section{Examples}

In this section we use Theorem 4.3 to establish the claims in Examples 1.13, 1.14, and to discuss Example 1.15 in detail.

The first claim in Example 1.13 follows from Theorem 4.3 or its corollaries since

$$
E^{\infty} \circ h=\left(E^{\infty}, E^{\infty} \circ h^{\prime}\right): \pi_{m}\left(S^{n}\right) \rightarrow \pi_{m-n}^{S} \oplus \pi_{m-2 n+1}^{S}
$$

(cf (4.1)ff) is injective here. Indeed, in the stable range $m \leq 2 n-2$, already $E^{\infty}$ is an isomorphism; when $m=2 n-1$ the needed injectivity follows from the exact EHP-sequence

$$
\mathbb{Z} \stackrel{\cdot\left[\iota_{n}, \iota_{n}\right]}{\longrightarrow} \pi_{2 n-1}\left(S^{n}\right) \stackrel{E^{\infty}}{\longrightarrow} \pi_{n-1}^{S}
$$

(cf [18, Chapter XII, (2.3) and (2.4)]) and from the fact that the classical Hopf invariant

$$
H: \pi_{2 n-1}\left(S^{n}\right) \stackrel{E^{\infty} \circ h_{1}^{\prime}}{\longrightarrow} \pi_{0}^{S} \equiv \mathbb{Z}
$$

(cf [18, Chapter XI, (8.17)]) takes the value 2 on $\left[\iota_{n}, \iota_{n}\right]$ (cf [18, Chapter XI, (2.5)]).

According to the generalized "Wecken theorem" 1.10 in [10], the minimum number MCC agrees always with $N_{0} \equiv N_{\infty}$ when $m<2 n-2$. The remaining claims in Example 1.13 follow from [14, Theorems 1.12, 1.27 and 1.29] and from [16, Theorems 1.13 and 1.16]. 
Next let us prove the claims in Example 1.14. When $n \geq 2$ is even and $m \leq n+3$, then $n \leq 2 n-1$ (and hence $E^{\infty} \circ h$ is injective by the preceding proof) or else $n=2$ and $m=4$ or 5 (and then already $E^{\infty}$ alone is injective (cf [17, Propositions 5.3, 5.6 and Theorem 14.1.i]). Thus again all Nielsen numbers agree among themselves, and also with MCC (by [14, Theorems 1.12 and (the last claim in) 1.19]).

In view of Theorem 4.3 suspensions of the Hopf-Hilton homomorphism $h$ (cf (4.1)ff) play possibly a decisive role also in arbitrary dimensions $m, n$.

Lemma 5.1 (\# $\left.\operatorname{Ker}\left(E^{r} \circ h\right)\right)_{r=0,1, \ldots}$ is a nondecreasing sequence of finite integers that are at most \# $\pi_{m}\left(S^{n}\right)$. In fact, we have more: if $\# \pi_{m}\left(S^{n}\right)=\infty$, then $E^{\infty} \circ h$ is injective and \# $\operatorname{Ker}\left(E^{r} \circ h\right)=1$ for all $r \geq 0$.

The second claim is obvious when $m=n$ and was established in the preceding discussion when $m=2 n-1$ and $n \equiv 0(2)$.

Next we compare the Nielsen number functions $N_{r}$ and $N_{r+1}, r \geq 0$. To distinguish them, we define

$$
\#_{r}^{i}(m, Y):=\#\left\{\left(\left[f_{1}\right],\left[f_{2}\right]\right) \in\left(\pi_{m}(Y)\right)^{2} \mid N_{r}\left(f_{1}, f_{2}\right)=i\right\} \in\{0,1,2, \ldots, \infty\}
$$

for $i=0,1, \ldots$. These cardinalities sum up to the square of $\# \pi_{m}(Y)$ and vanish when $i \notin\left\{0,1, k:=\# \pi_{1}(Y)\right\}$ (cf Proposition 1.12). Clearly, $\#_{r}^{0}(m, Y) \leq \#_{r+1}^{0}(m, Y)$ and $\#_{r}^{k}(m, Y) \geq \#_{r+1}^{k}(m, Y)$.

Proposition 5.3 Let $m, Y=S^{n} / G$ and $r$ be as in Theorem 4.3. Then the following conditions are equivalent:

(i) $N_{r} \equiv N_{r+1}\left(\right.$ ie, $N_{r}\left(f_{1}, f_{2}\right)=N_{r+1}\left(f_{1}, f_{2}\right)$ for all $f_{1}, f_{2}: S^{m} \rightarrow Y$ )

(ii) $\# \operatorname{Ker}\left(E^{r} \circ h\right)=\# \operatorname{Ker}\left(E^{r+1} \circ h\right)(\mathrm{cf}(4.1) f f)$

(iii) $\#_{r}^{0}(m, Y)=\#_{r+1}^{0}(m, Y)$

(iv) $\#_{r}^{i}(m, Y)=\#_{r+1}^{i}(m, Y)$ for all $i=0,1, \ldots$

In particular, when comparing Nielsen number functions $N_{r}, N_{r^{\prime}}, 0 \leq r, r^{\prime} \leq \infty$, it suffices to count how often they vanish.

Proof If condition (ii) is satisfied then $\operatorname{Ker}\left(E^{r} \circ h\right)=\operatorname{Ker}\left(E^{r+1} \circ h\right)$ by Lemma 5.1 and $E$ is injective, when restricted to $\operatorname{Im}\left(E^{r} \circ h\right)$. Hence the criteria for $N_{r}$ and $N_{r+1}$ in Theorem 4.3 agree and condition (i) holds. In turn this implies (iv) and (iii). 
On the other hand, if (ii) is not satisfied, then $\pi_{m}\left(S^{n}\right)$ is finite by Lemma 5.1 and contains a class $[\tilde{f}]$ such that

$$
E^{r} \circ h([\tilde{f}]) \neq 0=E^{r+1} \circ h([\tilde{f}]) .
$$

Thus according to Theorem 4.3,

$$
N_{r}((p \circ)[\tilde{f}], 0) \neq 0=N_{r+1}((p \circ)[\tilde{f}], 0)
$$

and therefore $\#_{r}^{0}(m, Y)<\#_{r+1}^{0}(m, Y)$. This completes the proof.

Next we compute the cardinalities $\#_{r}^{i}(m, Y)(\mathrm{cf}(5.2))$ in a particularly simple special case.

Lemma 5.4 Given $m, n \geq 2, n$ even, assume that $h^{\prime} \equiv 0$ on $\pi_{m}\left(S^{n}\right)\left(\operatorname{cf}\left(4.1^{\prime}\right)\right)$. Consider the iterated suspension homomorphism $E^{r}: \pi_{m}\left(S^{n}\right) \rightarrow \pi_{m+r}\left(S^{n+r}\right)$, and the (finite) cardinality $Q_{r}:=\#\left\{\alpha \in E^{r}\left(\pi_{m}\left(S^{n}\right)\right) \mid 2 \alpha=0\right\}, 0 \leq r \leq \infty$. If $Y=S^{n} / \mathbb{Z}_{2}$ as in Theorem 4.3, then

$$
\begin{aligned}
& \#_{r}^{0}(m, Y)=Q_{r} \cdot\left(\# \operatorname{Ker} E^{r}\right)^{2}, \\
& \#_{r}^{1}(m, Y)=2 \cdot\left(\left(\# E^{r}\left(\pi_{m}\left(S^{n}\right)\right)-Q_{r}\right) \cdot\left(\# \operatorname{Ker} E^{r}\right)^{2},\right. \\
& \#_{r}^{2}(m, Y)=\left(\#\left(\pi_{m}\left(S^{n}\right)\right)\right)^{2}-\#_{r}^{0}(m, Y)-\#_{r}^{1}(m, Y) .
\end{aligned}
$$

(All these cardinalities are finite except when $m=n$; in this case $\#_{r}^{0}(m, Y)=1$, but $\left.\#_{r}^{1}(m, Y)=\#_{r}^{2}(m, Y)=\infty\right)$.

In particular, the number of pairs ([ $\left.\left.f_{1}\right],\left[f_{2}\right]\right), f_{i}: S^{m} \rightarrow S^{n} / \mathbb{Z}_{2}, i=1,2$, such that $N_{0}\left(f_{1}, f_{2}\right)=0$, is equal to $Q_{0}$, ie, to the number of elements of order less than or equal to 2 in $\pi_{m}\left(S^{n}\right)$.

Proof Our assumption $h^{\prime} \equiv 0$ simplifies the criteria in Theorem 4.3 considerably and implies also that $(-\iota) \circ\left[\tilde{f}_{2}\right]=-\left[\tilde{f}_{2}\right](\operatorname{cf}[18$, Chapter XI, (8.12)]). Thus the set of pairs $\left(\left[f_{1}\right],\left[f_{2}\right]\right) \in \pi_{m}(Y)^{2}$ such that $N_{r}\left(f_{1}, f_{2}\right)=0$ (or $N_{r}\left(f_{1}, f_{2}\right)=1$, resp.) is characterized by the following conditions:

(i) $E^{r}\left(\left[\tilde{f}_{2}\right]\right)$ is an element of order at most 2, or not, resp., in $E^{r}\left(\pi_{m}\left(S^{n}\right)\right)$.

(ii) $\left[\tilde{f}_{1}\right] \in\left(E^{r}\right)^{-1}\left\{ \pm E^{r}\left(\left[f_{2}\right]\right)\right\}$.

The "number" of elements $\left[f_{2}\right] \in \pi_{m}(Y)$ satisfying condition (i) is $Q_{r} \cdot \# \operatorname{Ker} E^{r}$, and $\left(\left(\# \operatorname{Im} E^{r}\right)-Q_{r}\right) \cdot \# \operatorname{Ker} E^{r}$, respectively. Each such $\left[f_{2}\right]$ can be paired with as many as $\#\left\{ \pm E^{r}\left[\tilde{f}_{2}\right]\right\} \cdot \# \operatorname{Ker} E^{r}$ homotopy classes $\left[f_{1}\right] \in \pi_{m}(Y)$ in order to satisfy condition (ii) also. 
If $m=n$, then $N_{r}\left(f_{1}, f_{2}\right)=0$, or 1 , or 2 , resp., according to whether $\left(\left[f_{1}\right],\left[f_{2}\right]\right)=0$, or lies in the remaining union of the diagonal and antidiagonal in $\pi_{m}(Y)^{2} \cong \mathbb{Z} \times \mathbb{Z}$, or outside of this union, respectively.

Finally let us apply Lemma 5.4 to the case where $m=16, Y=S^{6} / \mathbb{Z}_{2}$. We use the computations in Toda's book [17] (cf Theorems 7.3, 13.9 and the tables in Chapter $\mathrm{XIV)}$ as well as Serre's theorem (cf eg [17, (13.1)]).

\begin{tabular}{|c|c|c|c|}
\hline \multicolumn{2}{|r|}{$\pi_{16+r}\left(S^{6+r}\right)$} & $\# \operatorname{Ker}\left(E^{r}\right)$ & $E^{r}\left(\pi_{16}\left(S^{6}\right)\right)$ \\
\hline $\begin{array}{c}\pi_{16}\left(S^{6}\right) \\
E \Downarrow\end{array}$ & 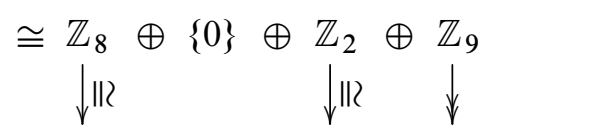 & 1 & $\mathbb{Z}_{8} \oplus \mathbb{Z}_{2} \oplus \mathbb{Z}_{9}$ \\
\hline $\begin{array}{c}\pi_{17}\left(S^{7}\right) \\
E \downarrow\end{array}$ & 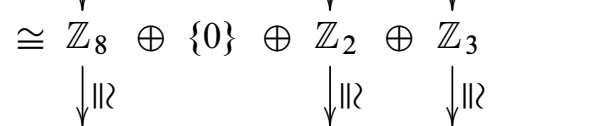 & 3 & $\mathbb{Z}_{8} \oplus \mathbb{Z}_{2} \oplus \mathbb{Z}_{3}$ \\
\hline $\begin{array}{c}\pi_{18}\left(S^{8}\right) \\
E \downarrow\end{array}$ & 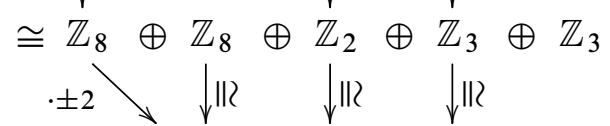 & 3 & $\mathbb{Z}_{8} \oplus \mathbb{Z}_{2} \oplus \mathbb{Z}_{3}$ \\
\hline $\begin{array}{c}\pi_{19}\left(S^{9}\right) \\
E \Downarrow\end{array}$ & $\cong \begin{array}{ccc}\mathbb{Z}_{8} & \oplus \mathbb{Z}_{2} & \oplus \mathbb{Z}_{3} \\
\downarrow & \left\|_{\| 2} \quad\right\|_{\| 2}\end{array}$ & 6 & $\mathbb{Z}_{4} \oplus \mathbb{Z}_{2} \oplus \mathbb{Z}_{3}$ \\
\hline $\begin{array}{c}\pi_{20}\left(S^{10}\right) \\
E \Downarrow\end{array}$ & $\begin{array}{ccc}\mathbb{Z}_{4} & \oplus \mathbb{Z}_{2} & \oplus \mathbb{Z}_{3} \\
\Downarrow & \|_{\| 2} & \downarrow \| 2\end{array}$ & 12 & $\mathbb{Z}_{2} \oplus \mathbb{Z}_{2} \oplus \mathbb{Z}_{3}$ \\
\hline $\begin{array}{c}\pi_{21}\left(S^{11}\right) \\
E \Downarrow\end{array}$ & $\begin{array}{lll}\mathbb{Z}_{2} & \oplus \mathbb{Z}_{2} & \oplus \mathbb{Z}_{3} \\
\Downarrow \| 2 & \|_{\| 2}\end{array}$ & 24 & $\mathbb{Z}_{2} \oplus \mathbb{Z}_{3}$ \\
\hline $\begin{array}{c}\pi_{22+j}\left(S^{12+j}\right) \\
j \geq 0\end{array}$ & $\mathbb{Z}_{2} \oplus \mathbb{Z}_{3}$ & 24 & $\mathbb{Z}_{2} \oplus \mathbb{Z}_{3}$ \\
\hline
\end{tabular}

Table 1: The suspension homomorphisms on the groups $\pi_{16+r}\left(S^{6+r}\right), r \geq 0$, as described by Toda [17]. The cyclic direct summands in the $i^{\text {th }}$ row, $i=1,2,3,4$, are generated by $v_{6+r} \circ \sigma_{9+r}, \sigma_{6+r} \circ v_{13+r}, \eta_{6+r} \circ \mu_{7+r}$ and $\beta_{1}(6+r)$, respectively.

In particular, the suspension homomorphism from $\pi_{15}\left(S^{5}\right)$ to $\pi_{16}\left(S^{6}\right)$ is both onto and injective. This implies not only that $h^{\prime} \equiv 0$ on $\pi_{16}\left(S^{6}\right)$, but also that $\operatorname{MC}\left(f_{1}, f_{2}\right)=$ $\operatorname{MCC}\left(f_{1}, f_{2}\right)=N_{0}\left(f_{1}, f_{2}\right)$ for all pairs $f_{1}, f_{2}: S^{16} \rightarrow S^{6} / \mathbb{Z}_{2}$ (cf Theorems 6.1 and 6.4 below, or else [11, Corollary 6.10 and Theorem 6.14] as well as [14, Theorem 1.19]). Moreover we can extract the explicit description of the groups $\pi_{16+r}\left(S^{6+r}\right), r=$ $0,1, \ldots$, and of the relevant suspension homomorphism as listed in Table 1. Eg, it follows from $[17,(4.4)$ and (7.19)] that

$$
E\left(v_{8} \circ \sigma_{11}\right)= \pm 2 \sigma_{9} \circ v_{16} .
$$


Now the data in Table 2 and the claims in Example 1.15 follow immediately from Lemma 5.4, Table 1 and Proposition 5.3.

\begin{tabular}{c|c|c|c|c|c}
$r$ & 0 & 1,2 & 3 & 4 & $r \geq 5$ \\
\hline$\#_{\operatorname{Ker}}\left(E^{r} \circ h\right)$ & 1 & 3 & 6 & 12 & 24 \\
$\#_{r}^{0}\left(16, S^{6} / \mathbb{Z}_{2}\right)$ & 4 & 36 & 144 & 576 & 1152 \\
$\#_{r}^{1}\left(16, S^{6} / \mathbb{Z}_{2}\right)$ & 280 & 792 & 1440 & 2304 & 4608 \\
$\#_{r}^{2}\left(16, S^{6} / \mathbb{Z}_{2}\right)$ & 20452 & 19908 & 19152 & 17856 & 14976 \\
$\#_{r}^{0}\left(16, S^{6}\right)$ & 144 & 432 & 864 & 1728 & 3456
\end{tabular}

Table 2: The value distributions of the Nielsen numbers $N_{r}$ for pairs of maps from $S^{16}$ to $S^{6} / \mathbb{Z}_{2}$ and to $S^{6}$. Here the suspension $E^{r} \circ h=$ $\left(E^{r}, 0\right): \pi_{16}\left(S^{6}\right) \rightarrow \pi_{r+16}\left(S^{r+6}\right)$ is equally relevant in both cases.

Moreover we conclude that there are precisely four "loose" pairs

$$
\left(\left[f_{1}\right],\left[f_{2}\right]\right) \in \pi_{16}\left(S^{6} / \mathbb{Z}_{2}\right)^{2}
$$

(ie, $\operatorname{MCC}\left(f_{1}, f_{2}\right)=0$ or, equivalently, $f_{1}$ and $f_{2}$ can be deformed away from one another); they have the form $\left(\left[f_{1}\right],\left[f_{2}\right]\right)=([p \circ \tilde{f}],[p \circ \tilde{f}])$ where $p$ denotes the projection and $[\tilde{f}]$ lies in the subgroup

$$
\mathbb{Z}_{2}\left(4 v_{6} \circ \sigma_{9}\right) \oplus \mathbb{Z}_{2}\left(\eta_{6} \circ \mu_{7}\right) \subset \pi_{16}\left(S^{6}\right) \cong \mathbb{Z}_{8} \oplus \mathbb{Z}_{2} \oplus \mathbb{Z}_{9}
$$

(compare [17, Theorem 7.3]).

Similarly, according to Theorem 4.3

$$
\#_{r}^{0}\left(16, S^{6}\right)=\# \pi_{16}\left(S^{6}\right) \cdot \# \operatorname{Ker} E^{r} .
$$

Therefore Table 1 (together with [11, 6.10 and 6.14]) also yields the claims in:

Example 5.5 $\left(m=16, Y=S^{6}\right)$ Here there are again five distinct Nielsen numbers (as in Example 1.15):

$$
\mathrm{MC} \equiv \mathrm{MCC} \equiv N_{0}, \quad N_{1} \equiv N_{2}, \quad N_{3}, \quad N_{4} \quad \text { and } \quad N_{5} \equiv \cdots \equiv N_{\infty} .
$$

They take only the values $i=0$ and 1 . The precise value distribution is given in Table 2. (Note that $\left.\#_{r}^{0}\left(16, S^{6}\right)+\#_{r}^{1}\left(16, S^{6}\right)=\left(\# \pi_{16}\left(S^{6}\right)\right)^{2}=20736\right)$.

Moreover, precisely the 144 pairs of the form $([\tilde{f}],-[\tilde{f}]),[\tilde{f}] \in \pi_{16}\left(S^{6}\right)$, are loose; unless $2[\tilde{f}]=0$ they do not project to loose pairs in $S^{6} / \mathbb{Z}_{2}$ (compare Example 1.15). 


\section{The minimum numbers $\mathrm{MC}$ and $\mathrm{MCC}$}

What can we say about the minimum numbers of coincidence points and coincidence path-components for maps into spherical space forms, once the Nielsen numbers - or at least $N_{0}$ - are understood?

Theorem 6.1 Let the finite group $G$ act smoothly and freely on $S^{n}$ and consider (basepoint preserving) maps

$$
f_{1}, f_{2}: S^{m} \rightarrow Y=S^{n} / G, \quad m, n \geq 1 .
$$

When $Y \cong S^{n}$ then $\operatorname{MCC} \equiv N_{0}$ (ie, $\operatorname{MCC}\left(f_{1}, f_{2}\right)=N_{0}\left(f_{1}, f_{2}\right)$ for all $\left.f_{1}, f_{2}\right)$.

When $\# G \geq 2$ then $\mathrm{MCC} \equiv N_{0}$ if and only if the "Wecken condition"

$$
0=\partial\left(\pi_{m}\left(S^{n}\right)\right) \cap \operatorname{Ker}\left(E: \pi_{m-1}\left(S^{n-1}\right) \rightarrow \pi_{m}\left(S^{n}\right)\right)
$$

holds; here $\partial:=\partial_{S^{n}}$ denotes the boundary homomorphism in the exact homotopy sequence of the tangent sphere bundle $S T\left(S^{n}\right)$ fibred over $S^{n}$ (as in Proposition 1.12).

Clearly condition (6.2) is satisfied when $n$ is odd, or $n=2$, or $m<n$, or $m<2 n-2$ (the "stable range"). But it can already fail to hold when $m=2 n-2$ or $m=2 n-1$. This explains the appearance of the Kervaire invariant and of the mod 4 Hopf invariant in the criteria in Example 1.13. For information concerning the next six nonstable dimension settings, see [16]. For the many geometric consequences of (a possible failure of) condition (6.2), see eg [14, Corollary 1.21].

Theorem 6.1 follows from [14, Corollary 1.20], except when $Y$ is a sphere. But if $Y=S^{n}$ and $a^{*}:\left(S^{n}, y_{0}\right) \rightarrow\left(S^{n}, y_{0}\right)$ is freely homotopic to the antipodal map and $[f]:=\left[f_{1}\right]-a_{*}^{\cdot}\left[f_{2}\right]$, then

$$
\operatorname{MCC}\left(f_{1}, f_{2}\right)=\operatorname{MCC}\left(f, y_{0}\right)=N_{0}\left(f, y_{0}\right)=N_{0}\left(f_{1}, f_{2}\right) .
$$

Indeed, if $n \geq 2$ as well, then

$$
\left(\left[f_{1}\right],\left[f_{2}\right]\right)=\left([f], y_{0}\right)+\left(a_{*}^{\cdot}\left[f_{2}\right],\left[f_{2}\right]\right) \quad \text { and } \operatorname{MCC}\left(a \circ f_{2}, f_{2}\right)=0 ;
$$

moreover, $\operatorname{MCC}\left(f, y_{0}\right) \leq 1$ vanishes precisely when $[f]=0$ or, equivalently, when $N_{0}\left(f, y_{0}\right)=0$ (since by construction $\omega^{\#}\left(f, y_{0}\right) \in \pi_{m}\left(S^{n} \wedge\left(\Omega S^{n}\right)^{+}\right)$determines $\left.[f] \in \pi_{m}\left(S^{n}\right)\right)$.

If $Y=S^{1}$, then our claim follows from Theorem 1.13 in [10]. This completes the proof of our Theorem 6.1. 
Once the minimum number MCC of coincidence path-components is determined, what about the minimum number MC of coincidence points? The answer for spherical space forms involves Hopf invariants in a decisive way. Indeed, let

$$
\underline{h}^{\prime}: \pi_{m-1}\left(S^{n-1}\right) \rightarrow \pi_{m-1}\left(S^{2 n-3}\right) \oplus \pi_{m-1}\left(S^{3 n-5}\right)^{2} \oplus \pi_{m-1}\left(S^{4 n-7}\right)^{3} \oplus \cdots
$$

denote the total Hopf-Hilton homomorphism (which involves all basic Whitehead products and not just a selection as in $\left(4.1^{\prime}\right)$; note also the different dimensions here).

Theorem 6.4 Given maps $f_{1}, f_{2}: S^{m} \rightarrow Y=S^{n} / G, m, n \geq 1$, as in Theorem 6.1, we have:

(i) If $\operatorname{MC}\left(f_{1}, f_{2}\right)<\infty$, then $\operatorname{MC}\left(f_{1}, f_{2}\right)=\operatorname{MCC}\left(f_{1}, f_{2}\right)$.

(ii) If $n=1$ or $m<n$, then $\operatorname{MC}\left(f_{1}, f_{2}\right)=\operatorname{MCC}\left(f_{1}, f_{2}\right)<\infty$.

If $m, n \geq 2$, then

$$
\operatorname{MC}\left(f_{1}, f_{2}\right)<\infty \Longleftrightarrow[\tilde{f}] \in \begin{cases}E\left(\operatorname{Ker}\left(\underline{h}^{\prime}\right)\right) & \text { if } \# G \geq 3, \\ E\left(\pi_{m-1}\left(S^{n-1}\right)\right) & \text { if } \# G \leq 2 .\end{cases}
$$

Here $[\tilde{f}]:=\left[\tilde{f}_{1}\right]-\left[\tilde{f}_{2}\right] \in \pi_{m}\left(S^{n}\right)$, where $\left[\tilde{f}_{i}\right]$ is obtained by lifting $\left[f_{i}\right] \in \pi_{m}\left(Y, y_{0}\right)$, $i=1,2$.

Proof We may assume that $m \geq n$ since otherwise $\pi_{m}\left(S^{n}\right)=0$ and $\mathrm{MC} \equiv \mathrm{MCC} \equiv 0$. The same holds if $m>n=1$. If $m=n=1$ and we denote the mapping degree of $f_{i}: S^{1} \rightarrow S^{1}$ by $d^{0}\left(f_{i}\right), i=1,2$, and put $d^{0}:=d^{0}\left(f_{1} \cdot f_{2}^{-1}\right)=d^{0}\left(f_{1}\right)-d^{0}\left(f_{2}\right)$, then

$$
\operatorname{MC}\left(f_{1}, f_{2}\right)=\operatorname{MCC}\left(f_{1}, f_{2}\right)=\left|d^{0}\left(f_{1}\right)-d^{0}\left(f_{2}\right)\right|<\infty
$$

since $f_{1} \cdot f_{2}^{-1}$ is homotopic to the map $z \rightarrow z^{d^{0}}, z \in S^{1}$, whose roots of unity belong to pairwise different Nielsen classes.

If $m>n=2$ and $\operatorname{MCC}\left(f_{1}, f_{2}\right)<\infty$, then $\operatorname{MC}\left(f_{1}, f_{2}\right)=\operatorname{MCC}\left(f_{1}, f_{2}\right)=0$ since each isolated coincidence point has an "index" in $\pi_{m-1}\left(S^{n-1}\right)=0$ and hence may be eliminated by small deformations (cf $[11,(28)]$ ). If $m=n=2$, then claim (i) follows from [9, Theorem 4.0].

Now we can deduce the full claim (ii) in our Theorem 6.4 from [11, Corollary 6.10] applied to $[f]:=\left[f_{1}\right]-\left[f_{2}\right]$ : just note that $\left|\operatorname{MC}\left(f_{1}, f_{2}\right)-\operatorname{MC}\left(\left[f_{1}\right]-\left[f_{2}\right], y_{0}\right)\right| \leq$ $\operatorname{MC}\left(f_{2}, f_{2}\right) \leq 1$ (cf [11, Proposition 6.2; 14, Theorem 1.19]). In order to also complete the proof of claim (i) we may assume that $m, n \geq 3$ and - in view of [11, Theorem 1.2] that $\operatorname{MC}\left(f_{1}, f_{2}\right) \leq \# G$. If $\operatorname{MC}\left(f_{1}, f_{2}\right) \neq \operatorname{MCC}\left(f_{1}, f_{2}\right)$, then obviously $N_{0}\left(f_{1}, f_{2}\right)<$ $\# G$ and hence (by Corollary 4.4) $f_{1} \sim f_{2}$ or $f_{1} \sim a \circ f_{2}$ and therefore $\operatorname{MC}\left(f_{1}, f_{2}\right)=$ $\operatorname{MCC}\left(f_{1}, f_{2}\right)=0$ or $1(\operatorname{cf}[14$, Theorem 1.19]). This is a contradiction. 
Since Hopf invariants vanish on suspended maps, we have the inclusions

$$
E^{2}\left(\pi_{m-2}\left(S^{n-2}\right)\right) \subset E\left(\operatorname{Ker}\left(\underline{h}^{\prime}\right)\right) \subset E\left(\pi_{m-1}\left(S^{n-1}\right)\right) \subset \operatorname{Ker} h^{\prime} \subset \pi_{m}\left(S^{n}\right),
$$

at least when $n \geq 3\left(\mathrm{cf}\left(4.1^{\prime}\right)\right.$ and (6.3)).

Corollary 6.6 Assume $n \geq 2$.

(i) If $\operatorname{MC}\left(f_{1}, f_{2}\right)<\infty$ then $h^{\prime}\left(\left[\tilde{f}_{1}\right]\right)=h^{\prime}\left(\left[\tilde{f}_{2}\right]\right)$. If $n$ is even and $m \leq 3 n-4$, then

$$
\operatorname{MC}\left(f_{1}, f_{2}\right)<\infty \Longleftrightarrow h^{\prime}\left(\left[\tilde{f}_{1}\right]\right)=h^{\prime}\left(\left[\tilde{f}_{2}\right]\right) .
$$

(ii) Now assume that $n$ is odd. Then

$$
\operatorname{MC}\left(f_{1}, f_{2}\right)=\operatorname{MCC}\left(f_{1}, f_{2}\right)= \begin{cases}\# G & \text { if } \widetilde{f}_{1} \nsim \tilde{f}_{2}, \\ 0 & \text { if } \widetilde{f}_{1} \sim \widetilde{f}_{2},\end{cases}
$$

provided $\left[\tilde{f}_{1}\right]-\left[\tilde{f}_{2}\right]$ lies in $E\left(\pi_{m-1}\left(S^{n-1}\right)\right)$ when $\# G \leq 2$ or in $E\left(\operatorname{Ker}\left(\underline{h}^{\prime}\right)\right)$ when $\# G \geq 3$. If this condition fails to hold, then $\operatorname{MCC}\left(f_{1}, f_{2}\right)=\# G$ but $\operatorname{MC}\left(f_{1}, f_{2}\right)$ is infinite.

Thus if $n$ is odd and $E\left(\operatorname{Ker}\left(\underline{h}^{\prime}\right)\right) \neq E\left(\pi_{m-1}\left(S^{n-1}\right)\right)$, the finiteness of $\operatorname{MC}\left(f_{1}, f_{2}\right)$ depends strongly on $\# G$.

Proof (i) Assume that $n$ is even and $m \leq 3 n-4$. Then $\# G \leq 2$ and $h^{\prime}$ fits into the exact EHP-sequence (cf [18, Chapter XII, 2.3])

$$
\pi_{m-1}\left(S^{n-1}\right) \stackrel{E}{\longrightarrow} \pi_{m}\left(S^{n}\right) \stackrel{H=h^{\prime}}{\longrightarrow} \pi_{m}\left(S^{2 n-1}\right) \longrightarrow \cdots .
$$

Indeed, $\pi_{m}\left(S^{2 m-1}\right)$ is stable and hence the Hopf-James invariant $H$ agrees with the Hopf-Hilton invariant $h^{\prime}$ (cf [4, Theorem 4.18]).

(ii) If $n$ is odd then $\operatorname{MCC}\left(f_{1}, f_{2}\right)=N_{0}\left(f_{1}, f_{2}\right)$ (cf Theorem 6.1) is described in Corollary 4.4(i); here a $\sim$ id.

Example 6.7 Let $m=2 n-2$ and $n=3,5$ or 9 , respectively. Then $\pi_{m}\left(S^{n}\right)$ is a cyclic group of order 2, 24 or 240 , resp., and

$$
E\left(\operatorname{Ker}\left(\underline{h}^{\prime}\right)\right)=2 \cdot \pi_{m}\left(S^{n}\right) \neq \pi_{m}\left(S^{n}\right)=E\left(\pi_{m-1}\left(S^{n-1}\right)\right) .
$$

If $\# G \leq 2$ then $\operatorname{MC}\left(f_{1}, f_{2}\right)<\infty$ for all maps $f_{1}, f_{2}: S^{m} \rightarrow S^{n} / G$. However, if $\# G \geq 3$ and $\left[\tilde{f}_{1}\right]-\left[\tilde{f}_{2}\right] \notin 2 \pi_{m}\left(S^{n}\right)$ then $\operatorname{MC}\left(f_{1}, f_{2}\right)=\infty$. 
Indeed here $\underline{h}^{\prime}: \pi_{m-1}\left(S^{n-1}\right) \rightarrow \mathbb{Z}$ coincides with the classical Hopf invariant homomorphism (cf [18, Chapter XI, 8.17]) and is onto. According to the first argument in Section 5 above the Freudenthal suspension epimorphism $E$ is injective when restricted to $\operatorname{Ker} \underline{h}^{\prime}$, ie, to the torsion subgroup of $\pi_{m-1}\left(S^{n-1}\right)$. An inspection of Toda's [17, Table I, page 186] now shows us that $E\left(\operatorname{Ker}\left(\underline{h}^{\prime}\right)\right)$ is a subgroup of index 2 of the cyclic group $E\left(\pi_{m-1}\left(S^{n-1}\right)\right)=\pi_{m}\left(S^{n}\right)$.

Finally let us take a look at maps into surfaces.

Example 6.8 $(m>n=2)$ Given maps $f_{1}, f_{2}$ from $S^{m}, m>2$, into any closed surface $Y$, we have

$$
\begin{array}{r}
\operatorname{MC}\left(f_{1}, f_{2}\right)= \begin{cases}\infty & \text { if } f_{1} \nsim f_{2}, \\
0 & \text { if } f_{1} \sim f_{2},\end{cases} \\
\operatorname{MCC}\left(f_{1}, f_{2}\right)=N_{0}\left(f_{1}, f_{2}\right)= \begin{cases}\# \pi_{1}(Y) & \text { if } f_{1} \nsucc f_{2}, \\
0 & \text { if } f_{1} \sim f_{2} .\end{cases}
\end{array}
$$

The same result holds for maps from $S^{m}$ into an $n$-dimensional spherical space form whenever $m, n \geq 2$ and $\pi_{m-1}\left(S^{n-1}\right)=0$, eg when $\left[f_{1}\right],\left[f_{2}\right] \in \pi_{22}\left(S^{10} / G\right) \cong \mathbb{Z}_{12}$.

Since all closed surfaces but $S^{2}$ and $\mathbb{R P}(2)$ are aspherical, these claims follow from:

Proposition 6.9 Assume that $\pi_{m-1}\left(S^{n-1}\right)=0$ where $m, n \geq 2$. Then we have for all maps $f_{1}, f_{2}: S^{m} \rightarrow S^{n} / G$ :

(i) If $f_{1} \sim f_{2}$, then $\operatorname{MC}\left(f_{1}, f_{2}\right)=\operatorname{MCC}\left(f_{1}, f_{2}\right)=N_{r}\left(f_{1}, f_{2}\right)=0$ for all $r=$ $0,1, \ldots, \infty$.

(ii) If $f_{1} \nsucc f_{2}$, then $\operatorname{MC}\left(f_{1}, f_{2}\right)=\infty$ but $\operatorname{MCC}\left(f_{1}, f_{2}\right)=N_{0}\left(f_{1}, f_{2}\right)=\# G$.

Proof If $\operatorname{MC}\left(f_{1}, f_{2}\right)<\infty$, the maps $f_{i}$ may be deformed until they have only isolated coincidence points. Each of these can be removed by a further local deformation since its index (very similar to the index of a vector field at an isolated zero; cf [11, (28)]) lies in $\pi_{m-1}\left(S^{n-1}\right)=0$. Thus $\operatorname{MC}\left(f_{1}, f_{2}\right)$ vanishes (and so do $\operatorname{MCC}\left(f_{1}, f_{2}\right)$ and the Nielsen numbers). According to Theorem 6.4 this happens precisely when $\left[f_{1}^{\prime}\right]=\left[f_{2}^{\prime}\right]$, where $f_{i}^{\prime}$ is any basepoint preserving map freely homotopic to $f_{i}, i=1,2$. Hence $f_{1} \sim f_{2}$; in turn, $\operatorname{MC}\left(f_{1}, f_{2}\right) \leq 1$ whenever $f_{1} \sim f_{2}$ (cf [14, Theorem 1.19]). The previous argument shows also that $\operatorname{MC}\left(f_{2}, f_{2}\right)=0$ even when $f_{1} \nsucc f_{2}$. Thus the pairs $\left(f_{1}, f_{2}\right)$ and $\left(\left[f_{1}^{\prime}\right]-\left[f_{2}^{\prime}\right], 0\right)$ have the same minimum and Nielsen numbers (cf $[11,6.2])$. Claim (ii) follows now from Theorem 6.1 and Corollary 4.4. 
It is a curious consequence of the last proof that each map $f: S^{m} \rightarrow S^{n}$ is homotopic to its composite $a \circ f$ with the antipodal map $a$ whenever $m, n \geq 2$ and $\pi_{m-1}\left(S^{n-1}\right)=0$. Indeed, clearly $\operatorname{MC}(f, a \circ f)=0$.

When the target manifold of our maps is not a spherical space form, certain finiteness conditions for the minimum number MC can still be expressed in terms of Hopf-Ganea invariants (cf Corollary 7.4 and Theorem 7.6 in [11]).

In the case when the target manifold is a (real, complex or quaternionic) projective space, a detailed discussion of minimum numbers and certain Nielsen numbers was carried out in $[13 ; 15]$.

\section{Appendix: The group $\pi_{m}\left(S^{q} \wedge(\Omega Y)^{+}\right)$and the partial sus- pension homomorphism $e$}

In our discussion of Nielsen numbers a central role is played by Thom spaces of the form $S^{q} \wedge\left((\Omega Y)^{+}\right)$. In this appendix we interpret these as fibers of appropriate fibrations. This allows us to study their homotopy groups, as well as suspension homomorphisms, which are important in coincidence theory.

Fix integers $m, q \geq 1$ and base points $\infty \in S^{q}, y_{0} \in Y$. Then the obvious collapsing map

$$
p_{2}: S^{q} \vee Y \rightarrow Y
$$

can be transformed (up to homotopy equivalences) into the fibration $\mathrm{ev}_{1}$ in the top line of the homotopy commutative diagram:

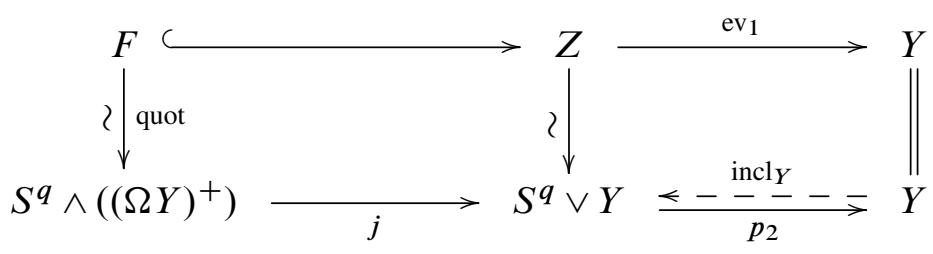

Here

$$
Z:=\left\{(x, \theta) \in\left(S^{q} \vee Y\right) \times Y^{I} \mid \theta(0)=p_{2}(x)\right\}
$$

is homotopy equivalent to $S^{q} \vee Y$ (via the first projection); the fiber map ev 1 evaluates the path $\theta$ at $1 \in I:=[0,1]$. The fiber

$$
F=\left\{(x, \theta) \in\left(S^{q} \vee Y\right) \times Y^{I} \mid \theta(0)=p_{2}(x), \theta(1)=y_{0}\right\}
$$


contains the contractible subspace $P=\{(x, \theta) \in F \mid x \in Y\}$, and the quotient map

$$
\text { quot: } F \rightarrow F / P=S^{q} \wedge\left((\Omega Y)^{+}\right)
$$

is a homotopy equivalence (compare Cornea [5, page 2769] and Koschorke [11, Lemma 7.1]). Its inverse (when composed with the fiber inclusion) yields the map

$$
j: S^{q} \wedge\left((\Omega Y)^{+}\right) \rightarrow S^{q} \vee Y .
$$

Obviously the collapsing map $p_{2}$ (cf (A.1)) allows a canonical right inverse. Thus the exact homotopy sequence of the fibration $\mathrm{ev}_{1}$ splits and takes the following form:

$$
0 \longrightarrow \pi_{m}\left(S^{q} \wedge\left((\Omega Y)^{+}\right)\right) \stackrel{j_{*}}{\longrightarrow} \pi_{m}\left(S^{q} \vee Y\right) \underset{\text { incl }_{Y} *}{\stackrel{p_{2 *}}{\longrightarrow}} \pi_{m}(Y) \longrightarrow 0
$$

We conclude:

Proposition A.8 The map $j$ (cf(A.6)) induces the isomorphism

$$
j_{*}: \pi_{m}\left(S^{q} \wedge\left((\Omega Y)^{+}\right)\right) \stackrel{\cong}{\longrightarrow} \operatorname{Ker}_{m, q}(Y):=\operatorname{Ker}\left(p_{2 *}: \pi_{m}\left(S^{q} \vee Y\right) \rightarrow \pi_{m}(Y)\right) .
$$

It will be useful to describe $j_{*}$ geometrically. Let $B^{q}(r)$ (and $\partial B^{q}(r)$, resp.), $r>0$, denote the compact ball (and sphere, resp.) of radius $r$ in $\mathbb{R}^{q}$, and use an (orientation preserving) standard identification $B^{q}(r) / \partial B^{q}(r)=S^{q}$. Given a base point preserving map

$$
u: S^{m}=\mathbb{R}^{m} \cup\{\infty\} \rightarrow\left(B^{q}(1) \times \Omega Y\right) /\left(\partial B^{q}(1) \times \Omega Y\right)=S^{q} \wedge\left((\Omega Y)^{+}\right)
$$

we may deform it until we have the following standard situation (as in Pontryagin-Thom theory): there is a smoothly embedded tubular neighbourhood $T_{3}:=B^{q}(3) \times C \subset \mathbb{R}^{m}$ of $C:=u^{-1}(\{0\} \times \Omega Y)$ such that

$$
u(x)= \begin{cases}{\left[\left(v, \theta_{u(c)}\right)\right]} & \text { if } x=(v, c) \in B^{q}(1) \times C, \\ \infty & \text { if } x \notin \stackrel{\circ}{B^{q}}(1) \times C .\end{cases}
$$

Here $\infty$ also denotes the base point of the Thom space $\left(\mathbb{R}^{q} \times \Omega Y\right) \cup\{\infty\}=S^{q} \wedge$ $\left((\Omega Y)^{+}\right)$, and $\theta_{u(c)}=u(0, c) \in \Omega Y(=\{0\} \times \Omega Y)$. Thus $u$ maps all normal slices $\stackrel{\circ}{B}^{q}(1) \times\{c\}$ in $T_{3}$ by the same diffeomorphism to the corresponding fibers $\mathbb{R}^{q} \times\left\{\theta_{u(c)}\right\}$ in the Thom space, $c \in C$.

Next consider the map

$$
u^{\prime}: S^{m} \rightarrow S^{q} \vee Y
$$


defined by

$$
\text { (A.10) } u^{\prime}(x) \begin{array}{ll}
{[v] \in B^{q}(1) / \partial B^{q}(1)=S^{q}} & \text { if } x=(v, c) \in B^{q}(1) \times C, \\
\theta_{u(c)}(\|v\|-1) \in Y & \text { if } x=(v, c) \in\left(B^{q}(2) \backslash B^{q}(1)\right) \times C, \\
\text { wedge point of } S^{q} \vee Y & \text { if } x \notin B^{q}(2) \times C .
\end{array}
$$

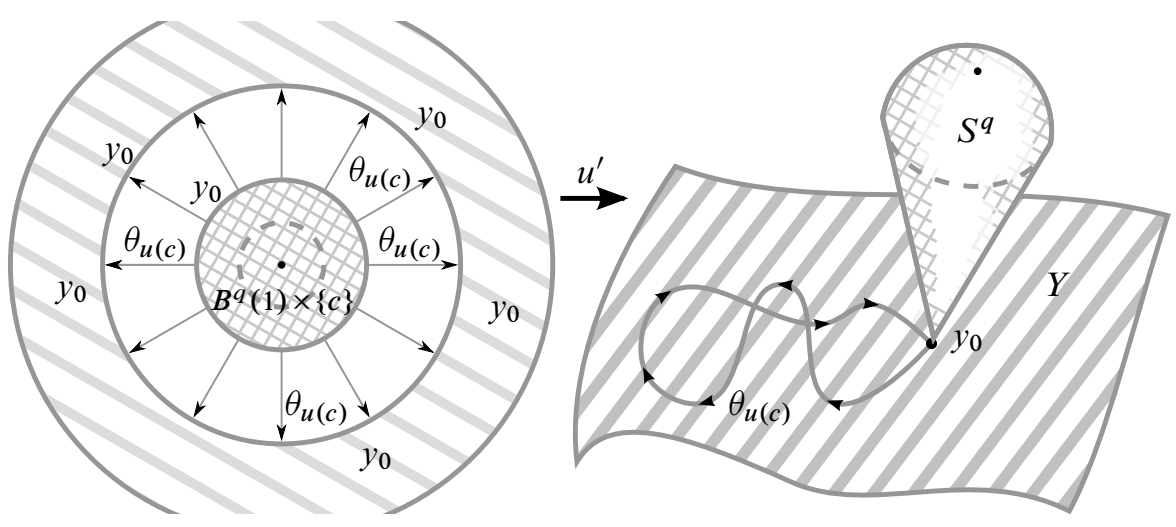

Figure 2: The image of $u^{\prime}$ on any normal slice $B^{q}(3) \times\{c\}, c \in C$.

Proposition A.11 $j_{*}([u])=\left[u^{\prime}\right]$

Proof We need only to lift $u^{\prime}$ to a map

$$
\tilde{u}^{\prime}: S^{m} \rightarrow F \subset Z
$$

such that quot $\circ \widetilde{u}^{\prime} \sim u$ (compare diagram (A.2)). In view of the standard form of $u$ and $u^{\prime}$, we can do so slice by slice. Given $c \in C$, let $x=(v, c)$ lie in the normal slice $B^{q}(3) \times\{c\}$ in the tubular neighbourhood $T_{3}$. Then we must find a path $\theta$ in $Y$ starting from $p_{2}\left(u^{\prime}(x)\right)$ and ending at $y_{0}$. If $\|v\| \leq 1$, then $p_{2}\left(u^{\prime}(x)\right)=y_{0}$ and we put $\theta=\theta_{u(c)}$ (compare (A.9) and (A.10)). If $1 \leq\|v\| \leq 2$, then $p_{2}\left(u^{\prime}(x)\right)=\theta_{u(c)}(\|v\|-1)$, and we define $\theta$ to be the path that first goes back to $y_{0}$ along $\theta_{u(c)}$ and then traverses the full loop $\theta_{u(c)}$. In particular, if $\|v\|=2$, then $\theta$ is $\theta_{u(c)}^{-1}$ followed by $\theta_{u(c)}$. We use the remaining parameter $2 \leq\|v\| \leq 3$ in the outer part $\left(B^{q}(3) \backslash B^{q}(2)\right) \times\{c\}$ of our normal slice to deform $\theta_{u(c)}^{-1} \cdot \theta_{u(c)}$ in $\Omega(Y)$ to the constant loop.

This procedure allows us to construct a continuous lifting of $u^{\prime}$ on the whole tubular neighbourhood $T_{3}=B^{q}(3) \times C$, and it can be extended trivially to all of $S^{m}$. All but the innermost part $B^{q}(1) \times C$ of $T_{3}$ gets mapped to $P$ (cf (A.5)) so that quot $\circ \widetilde{u}^{\prime}=u$ and $j_{*}([u])=(j \circ \text { quot })_{*}\left(\left[\tilde{u}^{\prime}\right]\right)=\left[u^{\prime}\right]$, as required. 
Next we construct a partial suspension homomorphism ${ }^{1}$

(A.12)

$$
e: \operatorname{Ker}_{m, q}(Y) \longrightarrow \operatorname{Ker}_{m+1, q+1}(Y)
$$

$$
\begin{array}{cc}
\cap & \cap \\
\pi_{m}\left(S^{q} \vee Y\right) & \pi_{m+1}\left(S^{q+1} \vee Y\right)
\end{array}
$$

(compare Proposition A.8), which suspends $S^{q}$ but leaves $Y$ unchanged. We use the same approach and notations as in (A.9) and (A.10).

Given $[w] \in \operatorname{Ker}_{m, q}(Y)$, we may assume that

$$
w: S^{m}=\mathbb{R}^{m} \cup\{\infty\} \rightarrow\left(B^{q}(1) / \partial B^{q}(1)\right) \vee Y=S^{q} \vee Y
$$

has the following standard form: there is a tubular neighbourhood $T_{1}=B^{q}(1) \times C \subset \mathbb{R}^{m}$ of $C=\{0\} \times C$ such that

(i) $w \mid T_{1}$ is the obvious composed projection from $B^{q}(1) \times C$ to $B^{q}(1) / \partial B^{q}(1)=$ $S^{q} \subset S^{q} \vee Y$, and

(ii) $w\left(S^{m} \backslash\left(\stackrel{\circ}{B^{q}}(1) \times C\right)\right) \subset Y \subset S^{q} \vee Y$.

Pick a base point preserving nullhomotopy $W: S^{m} \times[0,1] \rightarrow Y$ from $p_{2} \circ w$ to the constant map, and define

$$
e_{W} \mid: \mathbb{R}^{m} \times[-2,2] \rightarrow S^{q+1} \vee Y
$$

(i) on $T_{1} \times[-1,1]=\left(B^{q}(1) \times[-1,1]\right) \times C$ by the obvious projection to

$$
\left(B^{q}(1) \times[-1,1]\right) / \partial\left(B^{q}(1) \times[-1,1]\right)=S^{q+1} \subset S^{q+1} \vee Y,
$$

(ii) on $\left(\mathbb{R}^{m}-T_{1}\right) \times[-1,1]$ by the projection to $\mathbb{R}^{m}-T_{1}$, composed with the restricted map $w \mid$ into $Y \subset S^{q+1} \vee Y$,

(iii) for $\left(x, x_{m+1}\right) \in \mathbb{R}^{m} \times \mathbb{R}$ with $1 \leq\left|x_{m+1}\right| \leq 2$ by

$$
e_{W}\left(x, x_{m+1}\right)=W\left(x,\left|x_{m+1}\right|-1\right) \in Y \subset S^{q+1} \vee Y .
$$

These piecewise definitions fit well together and allow a trivial extension $e_{W}$ to all of $S^{m+1}=\left(\mathbb{R}^{m} \times \mathbb{R}\right) \cup\{\infty\}$.

The resulting homotopy class

$$
e([w]):=\left[e_{W}\right] \in \pi_{m+1}\left(S^{q+1} \vee Y\right)
$$

${ }^{1}$ After I had written this paper M Golasinski drew my attention to the work of H J Baues, who had introduced partial suspensions for suitable spaces $A, B$ and $Y$ (cf [1, Chapter 3]). My explicit geometric construction turns out to agree with Baues' homotopy theoretical definition for the case $A=S^{m}, B=S^{q}$. 
is independent of our choice of $W$. Indeed, given another nullhomotopy $W^{\prime}$ of $p_{2} \circ w$, let $\left[W^{-1} \cdot W^{\prime}\right] \in \pi_{m+1}(Y) \subset \pi_{m+1}\left(S^{q+1} \vee Y\right)$ be defined by concatenation; then

$$
\left[e_{W^{\prime}}\right]=-\left[W^{-1} \cdot W^{\prime}\right]+\left[e_{W}\right]+\left[W^{-1} \cdot W^{\prime}\right]=\left[e_{W}\right] .
$$

Similarly, $p_{2 *}\left(\left[e_{W}\right]\right)=0$, again due to the symmetry property of our construction with respect to the variable $x_{m+1}$.

Thus we obtain a well-defined partial suspension homomorphism $e$ as in (A.12). Clearly $e$ restricts to the full (standard) suspension on the subgroup $\pi_{m}\left(S^{q}\right)$ of $\pi_{m}\left(S^{q} \vee Y\right)$.

Remark A.14 The representation of $e([w])$ need not to be quite so specific as in $\left(1.13^{\prime}\right)$. Let

$$
\widehat{T}_{1}=B^{q+1}(1) \times C \hookrightarrow \mathbb{R}^{m} \times \mathbb{R}=\mathbb{R}^{m+1} \subset S^{m+1}
$$

be a tubular neighbourhood inclusion that extends the inclusion of $T_{1}=B^{q}(1) \times C$ into $\mathbb{R}^{m}=\mathbb{R}^{m} \times\{0\}$ and takes the last coordinate in $B^{q+1}$ to $x_{m+1}$. Also, let

$$
\widehat{W}: S^{m+1} \backslash \widehat{T}_{1} \rightarrow Y \subset S^{q+1} \vee Y
$$

be any map that extends $w \mid S^{m} \backslash T$, maps the boundary $\partial \widehat{T}_{1}$ of $\widehat{T}_{1}$ to the wedge-point, and satisfies the symmetry condition

$$
\widehat{W}\left(x, x_{m+1}\right)=\widehat{W}\left(x,-x_{m+1}\right)
$$

for all $\left(x, x_{m+1}\right) \in \mathbb{R}^{m} \times \mathbb{R} \subset S^{m+1},\left(x, x_{m+1}\right) \notin \widehat{T}_{1}$. Using a suitable ambient deformation of $\widehat{T}_{1}$ into $T_{1} \times[-1,1]$, it is not hard to see that the map

$$
e_{\widehat{W}}: S^{m+1} \rightarrow S^{q+1} \vee Y,
$$

defined by $\widehat{W}$ and the projection

$$
\widehat{T}_{1}=B^{q+1} \times C \rightarrow B^{q+1}(1) / \partial B^{q+1}(1)=S^{q+1} \subset S^{q+1} \vee Y,
$$

represents $e([w])$.

Theorem A.15 The partial suspension homomorphism $e$ has the following properties (where $E$ denotes (standard) full suspension homomorphisms and $m, m^{\prime}, q, q^{\prime} \geq 1$ ):

(a) Compatibility with the isomorphism $\boldsymbol{j}_{*}$ The diagram

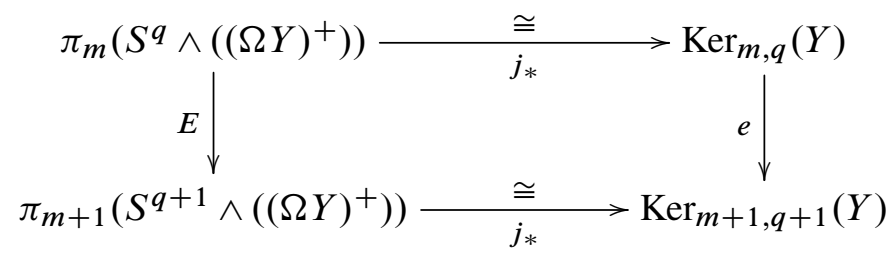


(cf Proposition A.8 and (A.12) ) commutes.

(b) Naturality

(a) Given a base point preserving map $g: S^{m^{\prime}} \rightarrow S^{m}$ and $[w] \in \operatorname{Ker}_{m, q}(Y)$, we have

$$
e([w \circ g])=(e([w])) \circ[E g] \in \operatorname{Ker}_{m^{\prime}+1, q+1}(Y) \text {. }
$$

(b) Given base point preserving maps $g_{1}: S^{q} \rightarrow S^{q^{\prime}}$ and $g_{2}: Y \rightarrow Y^{\prime}$ between manifolds, the map $g_{1} \vee g_{2}: S^{q} \vee Y \rightarrow S^{q^{\prime}} \vee Y^{\prime}$ induces the commuting diagram:

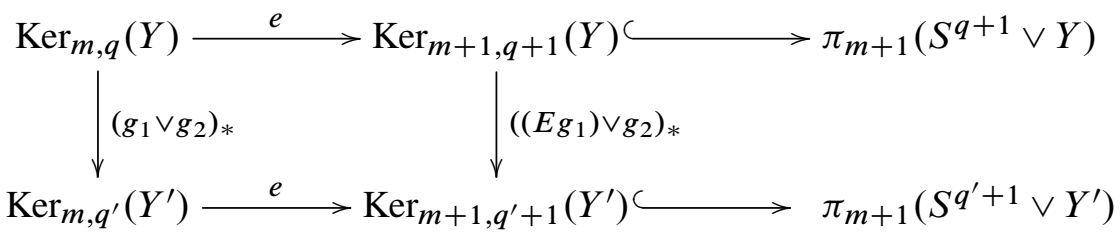

\section{(c) Compatibility with Whitehead products}

Given $\alpha \in \pi_{m}\left(S^{q} \vee Y\right)=\pi_{m}(Y) \oplus \operatorname{Ker}_{m, q}(Y)$ and $\beta \in \operatorname{Ker}_{m^{\prime}, q}(Y)$, we have:

(i) If $\alpha \in \pi_{m}(Y)$, then $e([\alpha, \beta])= \pm[\alpha, e(\beta)] \in \operatorname{Ker}_{m+m^{\prime}, q+1}(Y)$.

(ii) If $\alpha \in \operatorname{Ker}_{m, q}(Y)$, then $e([\alpha, \beta])=0$.

Proof Given $[u] \in \pi_{m}\left(S^{q} \wedge(\Omega Y)^{+}\right)$, pick a representative $u$ in standard form (as in (A.9) ), based on a map $g: C \rightarrow \Omega Y$ and on a framed embedding $C \subset \mathbb{R}^{m}$. Then we can represent the suspension $E([u])$ by a map $E u$ in standard form, based on the same $g$ and on the composite embedding $C \subset \mathbb{R}^{m} \subset \mathbb{R}^{m+1}$. Now compare the corresponding maps $u^{\prime}$ and $(E u)^{\prime}$ in standard form (cf (A.10) and (A.13) ) and apply Remark A.14 to $w:=u^{\prime}$. When we restrict $(E u)^{\prime}$ to the complement of the tubular neighbourhood $\widehat{T}_{1}$ of $C$ in $S^{m+1}$, we obtain a map $\widehat{W}$ as in Remark A.14. Thus

$$
\left(e \cdot j_{*}([u])=\right) \quad e\left(\left[u^{\prime}\right]\right)=\left[(E u)^{\prime}\right] \quad\left(=j_{*} \circ E([u])\right) .
$$

This establishes our first claim.

Naturality follows similarly from the way $e$ is defined or from Remark A.14.

For the proof of our third claim we use the geometric description of Whitehead products suggested eg by Chapter X, (7.1) or Figure 10.2 in [18]. Write

$$
\mathbb{R}^{m+m^{\prime}-1}=\mathbb{R}^{m-1} \times \mathbb{R} \times \mathbb{R}^{m^{\prime}-1}
$$

and let $S^{\prime} \subset \mathbb{R}^{m+m^{\prime}-1}$ denote the unit sphere (with center 0 ) of $\mathbb{R}^{m-1} \times \mathbb{R} \times\{0\}$, framed in the standard fashion by the outward pointing vector and $\mathbb{R}^{m^{\prime}-1}$. Similarly, 
let $S \subset\{0\} \times \mathbb{R} \times \mathbb{R}^{m^{\prime}-1}$ be the framed unit sphere (with center $e_{m}=(0,1,0) \in S^{\prime}$ ) of the normal space of $S^{\prime}$ at $e_{m}$. Also let

$$
T:=B^{m} \times S, T^{\prime}=B^{m^{\prime}} \times S^{\prime} \subset \mathbb{R}^{m+m^{\prime}-1}
$$

denote compact tubular neighbourhoods of $S$ and $S^{\prime}$, resp., parametrized compatibly with the framings and disjoint (but linked).

Now pick representatives $a, b$ in standard form (cf (A.4)) of the homotopy classes $\alpha, \beta \in \pi_{*}\left(S^{q} \vee Y\right)$. Define

$$
w_{a, b}: S^{m+m^{\prime}-1} \rightarrow S^{q} \vee Y
$$

on the tubular neighbourhood $T$ and $T^{\prime}$, resp., by composing $a$ and $b$, resp., with the obvious projections (eg compose $\alpha$ with $T=B^{m} \times S \rightarrow B^{m} / \partial B^{m}=S^{m}$ ), and on $S^{m+m^{\prime}-1}-\left(T \cup T^{\prime}\right)$ by the constant map. Then

$$
\left[w_{a, b}\right]= \pm[\alpha, \beta]
$$

and $w_{a, b}$ is again in standard form.

Now construct $e([b])=\left[e_{\widehat{W}}\right]$ as in Remark A.14 and consider the map

$$
\hat{T}^{\prime}=B^{m^{\prime}+1} \times S^{\prime} \longrightarrow S^{m^{\prime}+1} \stackrel{e_{\widehat{W}}}{\longrightarrow} S^{q} \vee Y,
$$

which extends $w_{a, b} \mid T^{\prime}$ to a tubular neighbourhood of $S^{\prime}$ in $\mathbb{R}^{m+m^{\prime}}$. If $a$ maps $S^{m}$ fully into $Y$, we can also extend $w_{a, b} \mid T$ to a tubular neighbourhood $\widehat{T}=B^{m} \times \widehat{S}$ of the unit sphere $\hat{S}$ (around $\left.e_{m}\right)$ in $\left(\{0\} \times \mathbb{R} \times \mathbb{R}^{m^{\prime}-1}\right) \times \mathbb{R} \subset \mathbb{R}^{m+m^{\prime}}$ by applying $a$ to each normal slice. We get a representative of $e([\alpha, \beta])$ (as in Remark A.14), which also represents $\pm[\alpha, e(\beta)]$. This proves the first part of claim (c).

If $\alpha \in \operatorname{Ker}_{m, q}(Y)$ we do not need all of $\hat{S}$, but we can extend both $w_{a, b} \mid T$ and $w_{a, b} \mid T^{\prime}$ to tubular neighbourhoods of $S, S^{\prime} \subset \mathbb{R}^{m+m^{\prime}-1}$ in $\mathbb{R}^{m+m^{\prime}}$. But these tubular neighbourhoods are not linked and can be isotoped to disjoint $x_{m+m^{\prime}}$-levels. Thus

$$
e([\alpha, \beta])=[0, e(\beta)]+[e(\alpha), 0]=0 .
$$

Corollary A.16 Let $[w] \in \pi_{*}\left(S^{q} \vee Y\right)$ be an iterated Whitehead product with factors in $\pi_{*}(Y)$ and with at least one factor purely in $\pi_{*}\left(S^{q}\right)$. If $[w]$ has precisely one factor $[v] \in \pi_{*}\left(S^{q}\right)$, then $\pm e([w])$ equals the same Whitehead product, but with $[v]$ replaced by the standard suspension $E([v]) \in \pi_{*}\left(S^{q+1}\right)$; otherwise $e([w])=0$.

Proof This follows by applying Theorem A.15(c) and the anticommutativity of Whitehead products repeatedly. 


\section{References}

[1] H J Baues, Obstruction theory on homotopy classification of maps, Lecture Notes in Mathematics 628, Springer, Berlin (1977) MR0467748

[2] R B S Brooks, On removing coincidences of two maps when only one, rather than both, of them may be deformed by a homotopy, Pacific J. Math. 40 (1972) 45-52 MR0341470

[3] R F Brown, Wecken properties for manifolds, from: "Nielsen theory and dynamical systems”, (C K McCord, editor), Contemp. Math. 152, Amer. Math. Soc. (1993) 9-21 MR1243467

[4] R F Brown, H Schirmer, Nielsen coincidence theory and coincidence-producing maps for manifolds with boundary, Topology Appl. 46 (1992) 65-79 MR1177164

[5] O Cornea, New obstructions to the thickening of $C W$-complexes, Proc. Amer. Math. Soc. 132 (2004) 2769-2781 MR2054804

[6] O Cornea, G Lupton, J Oprea, D Tanré, Lusternik-Schnirelmann category, Mathematical Surveys and Monographs 103, Amer. Math. Soc. (2003) MR1990857

[7] T Ganea, A generalization of the homology and homotopy suspension, Comment. Math. Helv. 39 (1965) 295-322 MR0179791

[8] P J Hilton, On the homotopy groups of the union of spheres, J. London Math. Soc. 30 (1955) 154-172 MR0068218

[9] J Jezierski, The least number of coincidence points on surfaces, J. Austral. Math. Soc. Ser. A 58 (1995) 27-38 MR1313883

[10] U Koschorke, Nielsen coincidence theory in arbitrary codimensions, J. Reine Angew. Math. 598 (2006) 211-236 MR2270573

[11] U Koschorke, Nonstabilized Nielsen coincidence invariants and Hopf-Ganea homomorphisms, Geom. Topol. 10 (2006) 619-666 MR2240900

[12] U Koschorke, Selfcoincidences and roots in Nielsen theory, J. Fixed Point Theory Appl. 2 (2007) 241-259 MR2372987

[13] U Koschorke, Minimizing coincidence numbers of maps into projective spaces, from: "The Zieschang Gedenkschrift", (M Boileau, M Scharlemann, R Weidmann, editors), Geom. Topol. Monogr. 14 (2008) 373-391 MR2484710

[14] U Koschorke, Minimum numbers and Wecken theorems in topological coincidence theory, I, J. Fixed Point Theory Appl. 10 (2011) 3-36 MR2825738

[15] U Koschorke, Nielsen numbers in topological coincidence theory, Topology Appl. 159 (2012) 3786-3796 MR2991953

[16] U Koschorke, D Randall, Kervaire invariants and selfcoincidences, Geom. Topol. 17 (2013) 621-638 MR3070513 
[17] H Toda, Composition methods in homotopy groups of spheres, Annals of Mathematics Studies 49, Princeton Univ. Press (1962) MR0143217

[18] G W Whitehead, Elements of homotopy theory, Graduate Texts in Mathematics 61, Springer, New York (1978) MR516508

Department Mathematik, Universität Siegen

Emmy Noether Campus, Walter-Flex-Str. 3, D-57068 Siegen, Germany

koschorke@mathematik. uni-siegen.de

Received: 6 June $2013 \quad$ Revised: 22 August 2013 
